\title{
WORLDWIDE GEOGRAPHICAL DISTRIBUTION OF BLACK SIGATOKA FOR BANANA: PREDICTIONS BASED ON CLIMATE CHANGE MODELS
}

\author{
Waldir Cintra de Jesus Júnior ${ }^{1 *}$; Ranolfo Valadares Júnior ${ }^{1}$; Roberto Avelino Cecílio ${ }^{1}$; Willian \\ Bucker Moraes $^{1}$; Francisco Xavier Ribeiro do Vale ${ }^{2}$; Fábio Ramos Alves ${ }^{1}$; Pierce Anderson \\ Paul $^{3}$ \\ ${ }^{l}$ UFES/CCA - Depto. de Produção Vegetal - Lab. de Fitopatologia, Alto Universitário, s/n. C.P. 16 - 29500-000 - \\ Alegre, ES - Brasil. \\ ${ }_{3}^{2}$ UFV/CCA - Depto. de Fitopatologia - 36570-000 - Viçosa, MG - Brasil. \\ 3 Ohio State University, Dept. of Plant Pathology - Wooster, Ohio - USA. \\ *Corresponding author <wcintra@yahoo.com>
}

\begin{abstract}
Global climatic changes will potentially influence plant diseases and the efficacy of their management options. One of the most likely impacts of climate change will be felt by the geographical distribution of plant diseases. Black Sigatoka is considered the most damaging and costly disease of banana. The socio-economic impact of this disease has continued to increase as the pathogen reaches new areas and the disease becomes more difficult to be controled. The objectives of this research were to compare the global geographical distribution of the disease based on maps elaborated using weather data representing: i) current and future periods (2020, 2050 and 2080), ii) Intergovernmental Panel on Climate Change scenarios A2 and B2, iii) predictions based on six different climate change models and the "multimodel ensemble" and, iv) individual months. The "multimodel ensemble" lead to a reduction in the variability of the simulations when compared to the results obtained using the individual models separately. The predictions suggested that, in the future, areas favorable for the development of the Black Sigatoka disease will decrease. This reduction will occur gradually and will be higher for the A2 than for the B2 scenario. Changes in the geographical distribution of the disease will occur from one month to another, with unfavorable areas becoming favorable and vice-versa. However, in spite of these changes, extensive areas will still continue to be favorable for the occurrence of Black Sigatoka.
\end{abstract}

Key words: Mycosphaerella fijiensis, Musa spp., global climate change

\section{DISTRIBUIÇÃO GEOGRÁFICA DA SIGATOKA NEGRA DA BANANEIRA ESTIMADA POR MODELOS DE MUDANÇAS CLIMÁTICAS GLOBAIS}

\begin{abstract}
RESUMO: As mudanças climáticas poderão alterar as doenças de plantas e afetar a eficácia das medidas de manejo. Um dos prováveis impactos será na distribuição geográfica das doenças. A Sigatoka Negra é considerada a principal doença da cultura da banana em decorrência dos danos causados e aumento do custo de manejo. O impacto sócio-econômico da doença continua aumentando, uma vez que a doença tem atingido novas áreas de plantio, tornando o manejo mais difícil. Este trabalho tem por objetivos comparar a distribuição geográfica da doença por meio da elaboração de mapas nas seguintes situações: a) clima atual e futuro (2020, 2050 e 2080), b) cenários A2 e B2 do Painel Intergovernamental de Mudanças Climáticas, c) predito por seis diferentes modelos de mudanças climáticas e pela média dos mesmos e, d) entre meses. Haverá redução das áreas favoráveis à doença no futuro, sendo que tal redução será mais acentuada no cenário A2 do que no B2 e gradativa para as décadas de 2020, 2050 e 2080. Predições efetuadas com o uso da média dos dados estimados pelos modelos permitiram redução na variabilidade da simulação em comparação com a predição gerada por cada modelo individualmente. Alterações na distribuição geográfica da doença ocorrerão entre meses, de modo que áreas consideradas desfavoráveis tornarse-ão favoráveis e vice-versa. Apesar disso, extensas áreas continuarão favoráveis ao desenvolvimento da Sigatoka Negra.

Palavras-chave: Mycosphaerella fijiensis, Musa spp., mudanças climáticas globais
\end{abstract}

Sci. Agric. (Piracicaba, Braz.), v.65, special issue, p.40-53, December 2008 


\section{INTRODUCTION}

Global climate change (GCC) is a major topic of discussion within both scientific and political forums. The Intergovernmental Panel on Climate Change (IPCC) is responsible for assessing information relevant to climate change and summarizing this information for policymakers and the public. GCC predictions are based on four scenarios (A1, A2, B1 and B2) that describe greenhouse gas emissions from potential resource using patterns, technological innovations, and demographics (IPCC, 2001).

Projections of future climate are made by computer models called Climate Change Models considering future greenhouse gas emissions simulated by each one of the four scenarios (A1, A2, B1 and B2). These models simulate climatic processes at different temporal and spatial scales to predict future changes at climate variables like air temperature, rainfall and relative humidity, among others.

A2 scenario describes a very heterogeneous world. The underlying theme is self-reliance and preservation of local identities. Fertility patterns across regions converge very slowly, which results in continuously increasing global population. Economic development is primarily regionally oriented and per capita economic growth and technological changes are more fragmented and slower than in other storylines (IPCC, 2001). The B2 scenario describes a world in which emphasis is put on local solutions for economic, social, and environmental sustainability. It is a world with continuously increasing global population at a rate lower than A2, intermediate levels of economic development, and less rapid and more diverse technological change than in the B1 and A1 storylines. While the scenario is also oriented toward environmental protection and social equity, it focuses on local and regional levels (IPCC, 2001).

The nature and magnitude of GCC will potentially influence plant diseases and the efficacy of their management options, impacting the productivity and sustainability of agricultural systems (Chakraborty et al., 1998). According to Chakraborty et al. (2000) one of the most likely impacts of climate change will be felt in the geographical distribution of plant diseases, with possible changes in the relative importance and spectrum of diseases and the emergence of new disease complexes. Modeling studies are essential to predict these alterations (Bergot et al., 2004; Scherm, 2004; Bourgeois et al., 2004), however methodology to use GCC models data on the prediction of global warming impacts on plant diseases has not yet been fully developed and still presents some lacks, like the use of the "multimodel emsemble", as it will be shown latter in this report.
Many studies were performed focusing plant diseases and global change. Carter et al. (1996) simulated climate changes in Finland concluding that increase in temperature will extend cereal cultivation areas in 2050. Moreover, the increase in the $\mathrm{CO}_{2}$ level probably will generate higher yield which will alter the geographical distribution of nematodes, extending to the north of the country with a higher number of nematode generation per year. In another study, the risk to the late blight of potato (Phytophthora infestans) was estimated to be higher over all areas at Finland. Similar results were observed in Europe for the nematodes Xiphinema spp. and Longidorus spp. (Boag et al., 1991) and for Phytophthora cinnamomi (Brasier \& Scott, 1994; Brasier et al., 1996). On other hand, these studies have focused on extratropical pathosystems, indicating a strong need for such studies on tropical areas.

Black Sigatoka (BS) (Mycosphaerella fijiensis Morelet) is considered one of the most damaging and costly diseases of banana (Marín et al., 2003). The socio-economic impact of BS continues to increase as the pathogen reaches new areas. As bananas are cultivated in more than 100 countries throughout the world and the impact of BS has also increased as it becomes more difficult to be controled (Marín et al., 2003), therefore the ability to assess worldwide geographical distribution of the worse disease of banana under climate change has practical implications on climatic zoning of the crop, establishment of agricultural government politics and adequate disease management.

The main objective of this research was to compare the monthly worldwide geographical distribution of BS at the current time and in the future (2020, 2050 and 2080 decades) using current climate data and six different GCC model predictions. A secondary objective was to compare the geographical distribution predicted by the use of each one of the six different climate change models with the one made by the use of "multimodel ensemble".

\section{MATERIAL AND METHODS}

Current climate data, average air temperature and air relative humidity, were used to elaborate maps of the geographical distribution of BS. Current climatic conditions were characterized based on information for the period between 1961 to 1990, available in a matrix format with cells of 10' on latitude ' 10' on longitude (New et al., 2002).

Data representing predicted variations in temperature and relative humidity for each month in the future were obtained from the IPCC website (IPCC, 2007). Future temperature data were calculated using 
predicted temperature variations obtained by six different climate change models: HadCM3 (Hadley Centre Coupled Model version 3), CSIROMk2 (Commonwealth Scientific and Industrial Research Organization GCM mark 2), CCSR/NIES (Centre for Climate Research Studies Model), ECHAM4 (European Centre Hamburg Model version 4), CGCM2 (Canadian Global Coupled Model version 2) and GFDL-R30 (Geophysical Fluid Dynamics Laboratory, R-30 resolution model) (IPCC, 2007). Only the HadCM3 model considered future variations in relative humidity, the other models assumed that relative humidity would remain constant or would only vary slightly in the future.

The chosen future scenarios were A2 and B2, with focus on the decades (or time slices) of 2020 (which comprises the periods of 2010 to 2039), 2050 (periods between 2040 and 2069) and 2080 (periods between 2070 and 2099) (IPCC, 2001). A1 and B1 scenarios were not used due to the fact that some climate change models don't use them to predict future variations on climate.

Predicted variations made by each model had a different spatial resolution (HadCM3: $3.75^{\circ} \times 2.5^{\circ}$, CSIRO-Mk2b: $5.625^{\circ} \times 3.214^{\circ}$, CCSR/NIES: $5.625^{\circ} \times$ 5.625 $5^{\circ}$ ECHAM4: $2.8125^{\circ} \times 2.8125^{\circ}$, CGCM2: $3.75^{\circ}$ $\times 3.75^{\circ}$ and GFDL-R30: $3.7^{\circ} \times 2.2^{\circ}$ ), so that climate variation data were resampled using the geographic information system (GIS) Idrisi $32^{\circledR}$ to generate maps with final spatial resolution of $10^{\prime}$ on latitude $\times 10^{\prime}$ on longitude. For each month, these maps were summed using Idrisi $32^{\circledR}$ (arithmetical operation), with the maps of current temperature and relative humidity to obtain future projections of these climatic variables.

There is no definitive consensus regarding which model is most appropriate for calculating values of the climatic variable for future scenarios (IPCC, 2007). So, maps of spatial distribution of BS were elaborated using the current climate and the following situations: i) Future air temperature estimated by each of the six climate change models and future relative humidity obtained from the HadCM3 model; ii) Future air temperature as the arithmetical mean of temperatures estimated using the six individual models and future relative humidity obtained from the HadCM3 model ("multimodel ensemble"). The methodology of map producing used was adapted of that one proposed by Hamada et al. (2006).

Based on the overlapping of maps of monthly average temperature and relative humidity, considering current and future period (2020, 2050 and 2080) for both scenarios (A2 and B2), new maps of disease distribution were elaborated using classes defined based on available epidemiological data on the effects of air temperature and relative humidity on the development of Black Sigatoka on banana (Table 1) (Meredith et al., 1973; Stover, 1983; Jacome et al., 1991; Jacome \& Schuh, 1992; Mouliom-Pefoura et al., 1996; Romero \& Sutton, 1997).

\section{RESULTS AND DISCUSSION}

From the total of 516 maps elaborated using monthly projections for current and future decades (2020, 2050 and 2080) in scenarios A2 and B2, only the results for the "multimodel ensemble" model are here shown (Figures 1 to 7 ). Complete results for simulations based on the six different climate change models and the "multimodel ensemble" are presented in Tables 2 to 4.

The intervals used in this study to characterize classes of favorability to BS were adequate because the obtained maps considering the current climate (Figure 1) were in agreement with data available in the literature (Mourichon et al., 1997; Carlier et al., 2000; Ghini et al., 2007). The pathogen produces conidia and ascospores and both play roles in the spread of the disease. Conidia are formed under conditions of high humidity, especially if there is a film of free water on the leaves. Ascospore release requires the presence of a rain or dew film of water that the pseudothecia imbibe and which results in the forcible ejection of the ascospores through the leaf boundary layer, from

Table 1- Classes of favorability for Black Sigatoka development defined as a function of temperature and relative humidity intervals.

\begin{tabular}{lccc}
\hline Favorability Classes & Description & Temperature intervals & Relative humidity intervals \\
\hline & & ${ }^{\circ} \mathrm{C}$ & $\%$ \\
1 & Highly favorable & 25 to 28 & $>90$ \\
2 & Favorable & 25 to 28 & 80 to 90 \\
3 & Relatively favorable & 20 to 25 or 28 to 35 & $>80$ \\
4 & Little favorable & 20 to 35 & 70 to 80 \\
$5 *$ & Unfavorable & $<20$ or $>35$ & $<70$ \\
\hline
\end{tabular}

*Favorability class 5 is defined if when any interval (temperature or relative humidity) occurs. For example: if temperature is between $20-35^{\circ} \mathrm{C}$ and relative humidity is less than $70 \%$ the area is classified at class 5 . 
Table 2- World area (\%) occupied by the classes of favorability for Black Sigatoka for each month at current date and 2020 (A2 and B2 scenarios) situations predicted by six climate change models and the multimodel ensemble.

\begin{tabular}{|c|c|c|c|c|c|c|c|c|c|c|c|c|c|c|c|c|}
\hline \multirow{2}{*}{$\overline{\text { Month }}$} & \multirow{2}{*}{\multicolumn{2}{|c|}{ Class Current }} & \multicolumn{7}{|c|}{$\mathrm{A} 2$} & \multicolumn{7}{|c|}{ B2 } \\
\hline & & & $\mathrm{CC}^{1}$ & $\mathrm{CG}^{2}$ & $\mathrm{CS}^{3}$ & $\mathrm{EC}^{4}$ & $\mathrm{GF}^{5}$ & $\mathrm{HC}^{6}$ & $\mathrm{AM}$ & $\mathrm{CC}^{1}$ & $\mathrm{CG}^{2}$ & $\mathrm{CS}^{3}$ & $\mathrm{EC}^{4}$ & $\mathrm{GF}^{5}$ & $\mathrm{HC}^{6}$ & AM \\
\hline \multirow{5}{*}{ January } & 1 & 0.085 & 0.003 & 0.003 & 0.003 & 0.003 & 0.003 & 0.003 & 0.003 & 0.004 & 0.004 & $4 \quad 0.004$ & 0.004 & 0.004 & $\begin{array}{ll}4 & 0.004\end{array}$ & 0.004 \\
\hline & 2 & 4.658 & 3.715 & 3.665 & 3.744 & 3.606 & 3.687 & 3.414 & 3.740 & 4.211 & 4.280 & $\begin{array}{ll}0 & 4.294\end{array}$ & 3.855 & 4.237 & $7 \quad 4.025$ & 4.283 \\
\hline & 3 & 2.092 & 1.216 & 1.238 & 1.145 & $5 \quad 1.294$ & $\begin{array}{l}4 \\
4\end{array}$ & 1.482 & 1.156 & 1.349 & 1.255 & $\begin{array}{ll}5 & 1.234\end{array}$ & 1.683 & 1.286 & 1.504 & 1.249 \\
\hline & 4 & 6.482 & 7.692 & 7.607 & 7.645 & 7.677 & 7.502 & 7.650 & 7.614 & 7.411 & 7.340 & $\begin{array}{ll}0 & 7.370\end{array}$ & 7.412 & 7.359 & 7.349 & 7.370 \\
\hline & 5 & 86.683 & 87.374 & 87.487 & 87.463 & 87.421 & 87.608 & 87.451 & 87.488 & 87.025 & 587.121 & 187.098 & $8 \quad 87.046$ & 87.115 & 587.119 & 87.095 \\
\hline \multirow{5}{*}{ February } & 1 & 0.211 & 0.007 & 0.007 & 0.007 & 0.007 & 0.007 & 0.007 & 0.007 & 0.009 & 0.009 & $\begin{array}{ll}9 & 0.009\end{array}$ & 0.009 & 0.009 & $\begin{array}{ll}9 & 0.009\end{array}$ & 0.009 \\
\hline & 2 & 4.590 & 4.412 & 4.384 & 4.472 & 4.291 & 4.369 & 4.291 & 4.430 & 4.359 & 4.371 & 14.362 & 4.061 & 4.276 & $\begin{array}{ll}6 & 4.194\end{array}$ & 4.357 \\
\hline & 3 & 1.719 & 1.137 & 1.152 & 1.046 & $5 \quad 1.240$ & 1.168 & 1.226 & 1.101 & 1.209 & 1.168 & $\begin{array}{ll}8 & 1.170\end{array}$ & 1.488 & 1.261 & 1.343 & 1.187 \\
\hline & 4 & 7.413 & 8.128 & 8.125 & 8.099 & 8.117 & 8.081 & 8.050 & 8.112 & 7.828 & 7.820 & $\begin{array}{ll}0 & 7.818\end{array}$ & 7.826 & 7.767 & 7.761 & 7.809 \\
\hline & 5 & 86.067 & 86.316 & 86.332 & 86.376 & 586.344 & 86.375 & 86.426 & 86.350 & 86.594 & 486.632 & 286.641 & 186.616 & 86.687 & 786.693 & 86.638 \\
\hline \multirow{5}{*}{ March } & 1 & 0.207 & 0.004 & 0.004 & 0.004 & 0.004 & 0.004 & 0.004 & 0.004 & 0.007 & 0.007 & 0.007 & 0.008 & 0.007 & 0.007 & 0.007 \\
\hline & 2 & 4.666 & 4.149 & 4.228 & 4.261 & 4.013 & 4.222 & 3.720 & 4.193 & 4.377 & 4.465 & $5 \quad 4.435$ & 3.952 & 4.458 & $\begin{array}{ll}8 \quad 4.130 \\
\end{array}$ & 4.361 \\
\hline & 3 & 1.396 & 1.194 & 1.096 & 1.051 & 1.298 & 1.071 & 1.578 & 1.117 & 1.313 & 1.163 & $\begin{array}{ll}3 & 1.259\end{array}$ & 1.690 & 1.202 & $\begin{array}{ll}2 & 1.514\end{array}$ & 1.294 \\
\hline & 4 & 8.274 & 8.909 & 8.881 & 8.899 & 8.850 & 8.931 & 8.844 & 8.888 & 8.495 & 8.459 & $\begin{array}{ll}9 & 8.538\end{array}$ & 8.487 & 8.475 & $\begin{array}{ll}5 & 8.451\end{array}$ & 8.493 \\
\hline & 5 & 85.457 & 85.744 & 85.791 & 85.785 & 85.836 & 58.771 & 85.854 & 85.798 & 85.808 & 855.906 & 685.760 & $\begin{array}{ll}085.863 \\
\end{array}$ & 85.857 & 785.897 & 85.845 \\
\hline \multirow{5}{*}{ April } & 1 & 0.159 & 0.006 & 0.004 & 0.003 & 0.005 & 0.005 & 0.005 & 0.005 & 0.006 & 0.004 & $4 \quad 0.005$ & 0.005 & 0.006 & 0.005 & 0.005 \\
\hline & 2 & 4.733 & 3.873 & 3.925 & 3.929 & 3.561 & 3.945 & 3.501 & 3.887 & 3.955 & 4.042 & 24.057 & 3.483 & 4.103 & 3.750 & 3.995 \\
\hline & 3 & 1.356 & 1.335 & 1.341 & 1.305 & 1.647 & 1.278 & 1.722 & 1.337 & 1.491 & 1.422 & 21.493 & 2.009 & 1.376 & 1.691 & 1.477 \\
\hline & 4 & 8.502 & 8.547 & 8.565 & 8.614 & 8.486 & 8.515 & 8.580 & 8.548 & 8.337 & 8.336 & $\begin{array}{ll}6 & 8.484\end{array}$ & 8.375 & 8.384 & $\begin{array}{ll}48.335 \\
\end{array}$ & 8.372 \\
\hline & 5 & 85.249 & 86.240 & 86.165 & 86.150 & 86.301 & 1 86.258 & 86.192 & 86.224 & 86.211 & 86.196 & 685.961 & 186.128 & 86.132 & 286.219 & 86.151 \\
\hline \multirow{5}{*}{ May } & 1 & 0.100 & 0.010 & 0.010 & 0.010 & 0.010 & 0.010 & 0.010 & 0.010 & 0.008 & 0.008 & $\begin{array}{ll}8 & 0.008\end{array}$ & 0.008 & 0.008 & $\begin{array}{ll}8 & 0.008\end{array}$ & 0.008 \\
\hline & 2 & 4.810 & 4.002 & 4.032 & 4.031 & 3.735 & 3.953 & 3.692 & 3.976 & 3.967 & 4.072 & $2 \quad 3.976$ & 3.569 & 4.023 & 3.591 & 3.926 \\
\hline & 3 & 1.605 & 1.578 & 1.545 & 1.555 & 1.851 & 1.619 & 1.872 & 1.602 & 1.610 & 1.500 & $\begin{array}{ll}0 & 1.607\end{array}$ & 2.017 & 1.567 & 1.987 & 1.656 \\
\hline & 4 & 8.664 & 8.280 & 8.320 & 8.267 & 8.256 & 8.190 & 8.167 & 8.246 & 8.274 & 8.251 & 18.300 & 8.213 & 8.226 & $\begin{array}{ll}6 & 8.187\end{array}$ & 8.250 \\
\hline & 5 & 84.821 & 86.130 & 86.093 & 86.137 & 86.148 & 86.228 & 86.258 & 86.166 & 86.142 & 286.170 & 086.110 & $\begin{array}{ll}086.193 \\
\end{array}$ & 86.176 & 686.228 & 86.160 \\
\hline \multirow{5}{*}{ June } & 1 & 0.067 & 0.035 & 0.032 & 0.032 & 0.035 & 0.032 & 0.034 & 0.033 & 0.035 & 0.035 & $\begin{array}{ll}5 & 0.036\end{array}$ & 0.041 & 0.037 & $\begin{array}{ll}7 & 0.035\end{array}$ & 0.036 \\
\hline & 2 & 4.806 & 4.316 & 4.390 & 4.390 & 3.906 & 4.252 & 4.173 & 4.299 & 4.391 & 4.461 & $1 \quad 4.517$ & 4.032 & 4.457 & 4.289 & 4.393 \\
\hline & 3 & 3.027 & 2.129 & 2.059 & 2.055 & 2.554 & 2.191 & 2.267 & 2.148 & 2.068 & 1.988 & $\begin{array}{ll}8 & 1.938\end{array}$ & 2.437 & 1.987 & $\begin{array}{ll}7 & 2.165\end{array}$ & 2.062 \\
\hline & 4 & 7.416 & 7.328 & 7.424 & 7.243 & 7.381 & 7.257 & 7.286 & 7.332 & 7.465 & 7.393 & $\begin{array}{ll}3 & 7.407\end{array}$ & 7.400 & 7.384 & $4 \quad 7.365$ & 7.408 \\
\hline & 5 & 84.683 & 86.191 & 86.096 & 86.281 & 86.124 & +86.268 & 86.240 & 86.189 & 86.040 & 0 86.122 & 286.102 & 286.090 & 86.136 & 686.145 & 86.101 \\
\hline \multirow{5}{*}{ July } & 1 & 0.008 & 0.029 & 0.028 & 0.029 & 0.030 & 0.030 & 0.029 & 0.029 & 0.023 & 0.022 & 20.025 & 0.025 & 0.023 & $\begin{array}{ll}3 & 0.029\end{array}$ & 0.024 \\
\hline & 2 & 4.489 & 4.037 & 3.989 & 4.228 & 3.878 & 4.044 & 4.167 & 4.096 & 4.099 & 4.072 & 24.223 & 4.101 & 4.301 & $\begin{array}{ll}1 \quad 4.269\end{array}$ & 4.215 \\
\hline & 3 & 3.905 & 3.058 & 3.093 & 2.850 & 3.242 & 3.041 & 2.926 & 2.998 & 3.153 & 3.149 & 93.018 & 3.159 & 2.937 & $\begin{array}{ll}7 & 2.981\end{array}$ & 3.029 \\
\hline & 4 & 9.603 & 9.692 & 9.860 & 9.910 & 10.184 & +10.055 & 9.830 & 9.898 & 10.649 & 9.879 & $\begin{array}{ll}9 & 9.991\end{array}$ & $\begin{array}{ll}1 \quad 10.177\end{array}$ & 10.173 & 310.083 & 10.008 \\
\hline & 5 & 81.995 & 83.185 & 83.031 & 82.983 & 82.665 & 82.830 & 83.048 & 82.979 & 82.077 & 782.878 & 882.744 & 482.538 & 82.566 & 682.638 & 82.725 \\
\hline \multirow{5}{*}{ August } & 1 & 0.009 & 0.026 & 0.023 & 0.025 & 0.027 & $\begin{array}{l}7 \quad 0.027 \\
\end{array}$ & 0.028 & 0.026 & 0.019 & 0.020 & $\begin{array}{ll}0 & 0.019\end{array}$ & 0.021 & 0.020 & $\begin{array}{ll}0 & 0.022\end{array}$ & 0.020 \\
\hline & 2 & 4.949 & 3.887 & 3.858 & 4.127 & 3.779 & 3.994 & 3.924 & 4.032 & 3.918 & 4.209 & $\begin{array}{l}9 \\
4.317\end{array}$ & 4.139 & 4.208 & $8 \quad 4.155$ & 4.283 \\
\hline & 3 & 3.725 & 3.093 & 3.106 & 2.844 & 3.218 & 2.997 & 3.058 & 2.948 & 3.559 & 3.225 & $5 \quad 3.150$ & 3.339 & 3.224 & $4 \quad 3.314$ & 3.179 \\
\hline & 4 & 9.718 & 10.503 & 10.242 & 10.520 & 10.894 & +10.630 & 10.569 & 10.537 & 10.189 & 9.583 & $\begin{array}{ll}310.024\end{array}$ & 410.129 & 10.041 & 19.946 & 9.955 \\
\hline & 5 & 81.599 & 82.490 & 82.770 & 82.483 & 82.083 & 82.352 & 82.421 & 82.457 & 82.314 & 482.964 & 482.490 & $\begin{array}{l}082.372 \\
\end{array}$ & 82.507 & 782.563 & 82.562 \\
\hline \multirow{5}{*}{ September } & 1 & 0.006 & 0.005 & 0.005 & 0.015 & 0.005 & 0.005 & 0.005 & 0.005 & 0.007 & 0.007 & $\begin{array}{ll}7 & 0.007\end{array}$ & 0.007 & 0.007 & $\begin{array}{ll}7 & 0.007\end{array}$ & 0.007 \\
\hline & 2 & 4.794 & 3.007 & 3.018 & 1.082 & 2.908 & 2.999 & 2.993 & 3.050 & 3.028 & 3.124 & $4 \quad 3.227$ & 2.939 & 3.091 & $1 \quad 2.988$ & 3.106 \\
\hline & 3 & 2.371 & 2.041 & 2.002 & 2.438 & 2.140 & 2.015 & 2.026 & 1.977 & 2.247 & 2.119 & $\begin{array}{l}9 \\
9\end{array}$ & 2.322 & 2.145 & $\begin{array}{ll}5 \quad 2.254 \\
\end{array}$ & 2.144 \\
\hline & 4 & 9.142 & 10.243 & 10.017 & 7.688 & 10.220 & 10.021 & 10.104 & 10.089 & 10.545 & 510.221 & 110.476 & $\begin{array}{ll}6 & 10.493\end{array}$ & 10.254 & 410.393 & 10.368 \\
\hline & 5 & 83.686 & 84.704 & 84.957 & 88.777 & 84.727 & 84.960 & 84.873 & 84.879 & 84.173 & 384.529 & 984.268 & $\begin{array}{l}8 \quad 84.238 \\
\end{array}$ & 84.503 & 384.358 & 84.375 \\
\hline
\end{tabular}


Table 2 - Continuation.

\begin{tabular}{|c|c|c|c|c|c|c|c|c|c|c|c|c|c|c|c|c|}
\hline \multirow{5}{*}{ October } & 1 & .006 & 0.005 & 0.005 & 0.005 & 0.005 & 0.005 & 0.005 & 0.005 & 0.007 & 0.006 & 0.006 & 0.006 & 0.006 & 0.006 & 0.006 \\
\hline & 2 & 4.523 & 2.492 & 2.540 & 2.527 & 2.318 & 2.396 & 2.361 & 2.451 & 2.687 & 2.797 & 2.787 & 2.427 & 2.778 & 2.453 & 2.717 \\
\hline & 3 & 1.496 & 1.017 & 0.938 & 0.954 & 1.180 & 1.103 & 1.133 & 1.039 & 1.173 & 1.021 & 1.055 & 1.418 & 1.041 & 1.371 & 1.112 \\
\hline & 4 & 7.621 & 9.081 & 8.806 & 8.793 & 8.907 & 9.065 & 9.021 & 8.922 & 8.643 & 8.340 & 8.390 & 8.485 & 8.325 & 8.417 & 8.429 \\
\hline & 5 & 86.354 & 87.404 & 87.711 & 87.721 & 87.590 & 87.431 & 87.480 & 87.583 & 87.489 & 87.836 & 87.762 & 87.664 & 87.849 & 87.752 & 87.736 \\
\hline \multirow{5}{*}{ November } & 1 & 0.022 & 0.005 & 0.005 & 0.005 & 0.005 & 0.005 & 0.005 & 0.005 & 0.006 & 0.006 & 0.006 & 0.006 & 0.006 & 0.006 & 0.006 \\
\hline & 2 & 4.112 & 2.984 & 3.080 & 2.982 & 2.634 & 2.961 & 2.470 & 2.904 & 3.072 & 3.494 & 3.389 & 2.683 & 3.310 & 2.738 & 3.210 \\
\hline & 3 & 1.516 & 1.358 & 1.223 & 1.340 & 1.707 & 1.363 & 1.867 & 1.425 & 1.684 & 1.231 & 1.345 & 2.079 & 1.418 & 2.004 & 1.533 \\
\hline & 4 & 7.375 & 8.108 & 7.904 & 8.010 & 8.002 & 7.995 & 8.045 & 8.011 & 7.768 & 7.597 & 7.664 & 7.697 & 7.645 & 7.712 & 7.682 \\
\hline & 5 & 86.975 & 87.545 & 87.788 & 87.664 & 87.653 & 87.676 & 87.613 & 87.655 & 87.470 & 87.672 & 87.597 & 87.534 & 87.621 & 87.541 & 87.570 \\
\hline \multirow{5}{*}{ December } & 1 & 0.049 & 0.008 & 0.008 & 0.008 & 0.008 & 0.008 & 0.008 & 0.008 & 0.009 & 0.009 & 0.009 & 0.009 & 0.009 & 0.009 & 0.009 \\
\hline & 2 & 4.269 & 3.494 & 3.473 & 3.484 & 3.228 & 3.430 & 2.988 & 3.412 & 3.319 & 3.438 & 3.410 & 3.190 & 3.385 & 2.924 & 3.339 \\
\hline & 3 & 1.795 & 1.052 & 1.014 & 1.014 & 1.270 & 1.060 & 1.500 & 1.084 & 1.276 & 1.073 & 1.135 & 1.382 & 1.150 & 1.599 & 1.198 \\
\hline & 4 & 7.480 & 8.416 & 8.241 & 8.329 & 8.288 & 8.218 & 8.254 & 8.290 & 8.386 & 8.326 & 8.438 & 8.365 & 8.365 & 8.351 & 8.380 \\
\hline & 5 & 86.407 & 87.030 & 87.264 & 87.165 & 87.206 & 87.284 & 87.251 & 87.205 & 87.010 & 87.155 & 87.008 & 87.056 & 87.091 & 87.118 & 87.075 \\
\hline
\end{tabular}

${ }^{1}$ CCSR/NIES (Centre for Climate Research Studies Model); ${ }^{2}$ CSIROMk2 (Commonwealth Scientific and Industrial Research Organization GCM mark 2); ${ }^{3}$ CGCM2 (Canadian Global Coupled Model version 2); ${ }^{4}$ ECHAM4 (European Centre Hamburg Model version 4); ${ }^{5}$ GFDL-R30 (Geophysical Fluid Dynamics Laboratory, R-30 resolution model); ${ }^{6} \mathrm{HadCM} 3$ (Hadley Centre Coupled Model version 3); ${ }^{7}$ Multimodel ensemble.

Table 3 - World area (\%) occupied by the classes of favorability for Black Sigatoka for each month at current date and 2050 (A2 and B2 scenarios) situations predicted by six climate change models and the multimodel ensemble.

\begin{tabular}{|c|c|c|c|c|c|c|c|c|c|c|c|c|c|c|c|c|}
\hline \multirow[b]{2}{*}{ Month } & \multirow{2}{*}{\multicolumn{2}{|c|}{ Class Current }} & \multicolumn{7}{|c|}{ A2 } & \multicolumn{7}{|c|}{ B2 } \\
\hline & & & $\mathrm{CC}^{1}$ & $\mathrm{CG}^{2}$ & $\mathrm{CS}^{3}$ & $\mathrm{EC}^{4}$ & $\mathrm{GF}^{5}$ & $\mathrm{HC}^{6}$ & $\mathrm{AM}$ & $\mathrm{CC}^{1}$ & $\mathrm{CG}^{2}$ & $\mathrm{CS}^{3}$ & $\mathrm{EC}^{4}$ & $\mathrm{GF}^{5}$ & $\mathrm{HC}^{6}$ & $\mathrm{AM}$ \\
\hline \multirow{5}{*}{ January } & 1 & 0.085 & 0.002 & 0.002 & 0.002 & 0.002 & 0.002 & 0.002 & 0.002 & 0.002 & 0.002 & 0.002 & 0.003 & 0.002 & 0.003 & 0.002 \\
\hline & 2 & 4.658 & 1.991 & 2.180 & 2.100 & 1.594 & 2.046 & 1.565 & 1.929 & 2.435 & 2.698 & 2.484 & 1.879 & 2.521 & 2.123 & 2.439 \\
\hline & 3 & 2.092 & 1.406 & 1.143 & 1.201 & 1.761 & 1.268 & 1.763 & 1.405 & 1.534 & 1.221 & 1.411 & 2.040 & 1.355 & 1.774 & 1.475 \\
\hline & 4 & 6.482 & 8.440 & 8.188 & 8.198 & 8.300 & 8.197 & 8.276 & 8.271 & 8.333 & 8.157 & 8.142 & 8.156 & 8.083 & 8.102 & 8.163 \\
\hline & 5 & 86.683 & 88.161 & 88.488 & 88.500 & 88.344 & 88.487 & 88.394 & 88.393 & 87.695 & 87.921 & 87.960 & 87.923 & 88.038 & 87.998 & 87.920 \\
\hline \multirow{5}{*}{ February } & 1 & 0.211 & 0.009 & 0.011 & 0.011 & 0.008 & 0.011 & 0.009 & 0.011 & 0.007 & 0.007 & 0.007 & 0.007 & 0.007 & 0.008 & 0.007 \\
\hline & 2 & 4.590 & 2.105 & 2.401 & 2.398 & 1.717 & 2.469 & 1.488 & 2.041 & 3.088 & 3.326 & 3.122 & 2.229 & 3.193 & 2.207 & 2.887 \\
\hline & 3 & 1.719 & 1.723 & 1.421 & 1.385 & 2.089 & 1.357 & 2.321 & 1.771 & 1.704 & 1.431 & 1.623 & 2.532 & 1.542 & 2.531 & 1.868 \\
\hline & 4 & 7.413 & 8.517 & 8.470 & 8.443 & 8.456 & 8.504 & 8.434 & 8.490 & 8.076 & 8.012 & 8.018 & 8.026 & 7.974 & 7.962 & 8.029 \\
\hline & 5 & 86.067 & 87.647 & 87.697 & 87.763 & 87.730 & 87.660 & 87.748 & 87.688 & 87.124 & 87.224 & 87.230 & 87.206 & 87.285 & 87.293 & 87.209 \\
\hline \multirow{5}{*}{ March } & 1 & 0.207 & 0.015 & 0.018 & 0.018 & 0.010 & 0.018 & 0.017 & 0.017 & 0.009 & 0.008 & 0.008 & 0.008 & 0.008 & 0.008 & 0.008 \\
\hline & 2 & 4.666 & 2.104 & 2.326 & 2.296 & 1.550 & 2.378 & 1.356 & 2.037 & 2.985 & 3.268 & 2.932 & 2.078 & 3.145 & 2.172 & 2.870 \\
\hline & 3 & 1.396 & 2.122 & 1.873 & 1.831 & 2.558 & 1.727 & 2.770 & 2.114 & 2.150 & 1.827 & 2.106 & 2.979 & 1.842 & 2.803 & 2.174 \\
\hline & 4 & 8.274 & 9.419 & 9.428 & 9.320 & 9.270 & 9.312 & 9.300 & 9.339 & 9.090 & 8.996 & 9.036 & 9.001 & 8.986 & 8.938 & 9.008 \\
\hline & 5 & 85.457 & 86.340 & 86.355 & 86.535 & 86.611 & 86.565 & 86.557 & 86.493 & 85.766 & 85.901 & 85.919 & 85.934 & 86.019 & 86.078 & 85.940 \\
\hline \multirow{5}{*}{ April } & 1 & 0.159 & 0.001 & 0.006 & 0.006 & 0.000 & 0.010 & 0.002 & 0.001 & 0.003 & 0.003 & 0.004 & 0.000 & 0.004 & 0.003 & 0.003 \\
\hline & 2 & 4.733 & 1.240 & 1.816 & 1.758 & 0.961 & 1.773 & 1.075 & 1.319 & 2.051 & 2.697 & 2.428 & 1.484 & 2.358 & 1.790 & 2.077 \\
\hline & 3 & 1.356 & 2.527 & 1.977 & 2.066 & 2.752 & 1.929 & 2.660 & 2.432 & 2.704 & 2.017 & 2.300 & 3.248 & 2.343 & 2.898 & 2.631 \\
\hline & 4 & 8.502 & 8.690 & 8.726 & 8.873 & 8.453 & 8.583 & 8.719 & 8.656 & 8.908 & 8.738 & 8.875 & 8.686 & 8.774 & 8.699 & 8.774 \\
\hline & 5 & 85.249 & 87.543 & 87.474 & 87.298 & 87.833 & 87.705 & 87.544 & 87.592 & 86.334 & 86.546 & 86.393 & 86.581 & 86.522 & 86.610 & 86.514 \\
\hline \multirow{5}{*}{ May } & 1 & 0.100 & 0.036 & 0.043 & 0.045 & 0.030 & 0.044 & 0.042 & 0.041 & 0.041 & 0.040 & 0.042 & 0.037 & 0.033 & 0.040 & 0.040 \\
\hline & 2 & 4.810 & 1.473 & 1.964 & 1.964 & 1.471 & 1.706 & 1.268 & 1.631 & 2.216 & 2.979 & 2.627 & 2.122 & 2.502 & 2.032 & 2.431 \\
\hline & 3 & 1.605 & 2.247 & 1.735 & 1.720 & 2.237 & 2.011 & 2.421 & 2.066 & 2.757 & 1.955 & 2.318 & 2.832 & 2.298 & 2.896 & 2.510 \\
\hline & 4 & 8.664 & 8.591 & 8.420 & 8.360 & 8.382 & 8.381 & 8.350 & 8.431 & 8.454 & 8.124 & 8.259 & 8.223 & 8.117 & 8.064 & 8.202 \\
\hline & 5 & 84.821 & 87.653 & 87.837 & 87.911 & 87.880 & 87.858 & 87.919 & 87.830 & 86.532 & 86.903 & 86.755 & 86.787 & 87.050 & 86.968 & 86.816 \\
\hline
\end{tabular}

Continue... 
Table 3 - Continuation.

\begin{tabular}{|c|c|c|c|c|c|c|c|c|c|c|c|c|c|c|c|c|}
\hline \multirow{5}{*}{ June } & 1 & 0.067 & 0.057 & 0.065 & 0.064 & 0.062 & 0.063 & 0.061 & 0.063 & 0.051 & 0.043 & 0.044 & 0.042 & 0.043 & 0.043 & 0,044 \\
\hline & 2 & 4.806 & 2.332 & 2.576 & 2.526 & 1.994 & 2.374 & 1.804 & 2.275 & 2.957 & 3.240 & 3.048 & 2.637 & 2.967 & 2.877 & 2.966 \\
\hline & 3 & 3.027 & 2.330 & 2.050 & 2.100 & 2.655 & 2.252 & 2.829 & 2.361 & 2.537 & 2.210 & 2.420 & 2.855 & 2.486 & 2.581 & 2.504 \\
\hline & 4 & 7.416 & 8.006 & 7.765 & 7.750 & 7.749 & 7.571 & 7.597 & 7.672 & .758 & 8.127 & 8.247 & 8.289 & 8.103 & 8.102 & 8.205 \\
\hline & 5 & 84.683 & 87.276 & 87.544 & 87.560 & 87.540 & 87.741 & 87.709 & 87.629 & 85.697 & 86.380 & 86.241 & 86.177 & 86.401 & 86.397 & 86.281 \\
\hline \multirow{5}{*}{ July } & 1 & 0.008 & 0.047 & 0.053 & 0.051 & 0.050 & 0.051 & 0.046 & 0.049 & 0.069 & 0.107 & 0.059 & 0.071 & 0.059 & 0.084 & 0.070 \\
\hline & 2 & 4.489 & 2.826 & 3.089 & 3.023 & 2.745 & 2.932 & 2.542 & 2.907 & 3.427 & 3.586 & 3.541 & 3.300 & 3.519 & 3.450 & 3.532 \\
\hline & 3 & 3.905 & 2.864 & 2.547 & 2.624 & 2.934 & 2.696 & 3.123 & 2.751 & 3.204 & 2.930 & 3.069 & 3.333 & 3.065 & 3.132 & 3.067 \\
\hline & 4 & 9.603 & 10.490 & 9.851 & 9.492 & 10.021 & 10.124 & 9.980 & 9.798 & 11.027 & 9.173 & 9.662 & 10.055 & 9.771 & 9.828 & 9.730 \\
\hline & 5 & 995 & 3.773 & 84.460 & 84.810 & 84.250 & 84.197 & 84.309 & 84.496 & 82.274 & 84.204 & 83.670 & 83.241 & 83.586 & 83.506 & 83.602 \\
\hline \multirow{5}{*}{ August } & 1 & 0.009 & 0.040 & 0.042 & 0.041 & 0.037 & 0.043 & 0.038 & 0.041 & 0.029 & 0.030 & 0.029 & 0.027 & 0.029 & 0.028 & 0.029 \\
\hline & 2 & 4.949 & 2.614 & 2.961 & 2.865 & 2.544 & 2.765 & 2.674 & 2.803 & 3.080 & 3.727 & 3.576 & 3.241 & 3.564 & 3.393 & 3.518 \\
\hline & 3 & 3.725 & 3.147 & 2.749 & 2.848 & 3.199 & 2.951 & 3.093 & 2.935 & 3.594 & 2.880 & 3.066 & 3.431 & 3.062 & 3.282 & 3.133 \\
\hline & 4 & 9 & & 9.4 & 9.803 & & & & & & 9.069 & & & & & 9.905 \\
\hline & 5 & 81.599 & 83.747 & 84.811 & 84.443 & 83.803 & 83.878 & 83.835 & 84.115 & 82.814 & 84.294 & 83.601 & 83.114 & 83.094 & 83.218 & 83.415 \\
\hline \multirow{5}{*}{ September } & 1 & 0.006 & 0.012 & 0.012 & 0.010 & 0.009 & 0.009 & 0.010 & 0.010 & 0.010 & 0.010 & 0.009 & 0.007 & 0.009 & 0.009 & 0.009 \\
\hline & 2 & 794 & 2.025 & 2.059 & 2.027 & 1.667 & 1.925 & 1.818 & 1.913 & 2.300 & 2.411 & 2.243 & 1.893 & 2.201 & 2.192 & 2.207 \\
\hline & 3 & 2 & 2.037 & 1.945 & 1.967 & 2.359 & 2.075 & 2.192 & & 2.069 & & 2.073 & & & & \\
\hline & 4 & 9.142 & 9.795 & 9.178 & 9.263 & 9.529 & 9.239 & 9.284 & 9.365 & 9.907 & 9.243 & 9.394 & 9.545 & 9.424 & 9.373 & 9.481 \\
\hline & 5 & 83.686 & 86.131 & 86.807 & 86.733 & 86.436 & 86.752 & 86.696 & 86.610 & 85.715 & 86.447 & 86.281 & 86.108 & 86.254 & 86.302 & 86.184 \\
\hline \multirow{5}{*}{ October } & 1 & & & 005 & 0.005 & 0.004 & .005 & 0. & .004 & 005 & 0.006 & 0.005 & 0.004 & 0.005 & 0.005 & .005 \\
\hline & 2 & 4.523 & 1.379 & 1.651 & 1.633 & 1.227 & 1.505 & 1.367 & 1.459 & 1.541 & 1.909 & 1.754 & & 1.784 & 1.724 & 1.671 \\
\hline & 3 & 1.496 & 1.664 & 1.326 & 1.351 & 1.782 & 1.480 & 1.635 & 1.547 & 1.669 & 1.211 & 1.411 & 1.883 & 1.343 & 1.411 & 1.488 \\
\hline & 4 & 7.621 & 8.067 & 7.328 & 7.250 & 7.426 & 7.335 & 7.574 & 7.482 & 7.770 & 7.028 & 7.106 & 7.291 & 7.201 & 7.229 & 7.271 \\
\hline & 5 & 86.354 & 88.885 & 89.690 & 89.761 & 89.560 & 89.675 & 89.419 & 89.508 & 89.014 & 89.846 & 89.724 & 89.511 & 89.666 & 89.630 & 89.565 \\
\hline \multirow{5}{*}{ November } & 1 & & & 0.005 & 0.009 & 0.005 & 0.005 & 0.012 & 0.005 & 0.006 & 0.007 & 0.007 & 0.006 & 0.007 & 0.007 & 0.006 \\
\hline & 2 & 4.112 & 1.446 & 1.733 & 1.678 & 1.342 & 1.587 & 1.513 & 1.554 & 1.944 & 2.159 & 1.979 & 1.671 & 1.923 & 1.957 & 1.939 \\
\hline & 3 & 1.516 & 1.604 & 1.288 & 1.355 & 1.702 & 1.455 & 1.521 & 1.489 & 1.595 & 1.352 & 1.544 & 1.867 & 1.599 & 1.562 & 1.586 \\
\hline & 4 & 7.375 & 7.481 & 7.207 & 7.236 & 7.238 & 7.250 & 7.322 & 7.297 & 7.830 & 7.596 & 7.685 & 7.714 & 7.658 & 7.676 & 7.693 \\
\hline & 5 & 86.975 & 89.464 & 89.767 & 89.722 & 89.713 & 89.703 & 89.632 & 89.655 & 88.625 & 88.887 & 88.786 & 88.742 & 88.813 & 88.798 & 88.775 \\
\hline \multirow{5}{*}{ December } & 1 & 0.049 & 0.007 & 0.007 & 0.007 & 0.007 & 0.007 & 0.009 & 0.007 & & 0.009 & 0.009 & 0.009 & 0.008 & 30.009 & 0.009 \\
\hline & 2 & 4.269 & 1.493 & 1.758 & 1.679 & 1.391 & 1.598 & 1.512 & 1.574 & 2.191 & 2.489 & 2.213 & 1.623 & 1.960 & 1.963 & 2.099 \\
\hline & 3 & 1.795 & 1.556 & 1.233 & 1.341 & 1.648 & 1.416 & 1.503 & 1.448 & 1.514 & 1.134 & 1.456 & 2.052 & 1.702 & 1.670 & 1.561 \\
\hline & 4 & 7.480 & 8.298 & 8.025 & 8.149 & 8.181 & 8.123 & 8.167 & 8.167 & 8.691 & 8.423 & 8.576 & 8.530 & 8.542 & 8.466 & 8.548 \\
\hline & 5 & 86.407 & 88.646 & 88.977 & 88.823 & 88.773 & 88.856 & 88.809 & 88.804 & 87.596 & 87.945 & 87.746 & 87.786 & 87.788 & 87.893 & 87.783 \\
\hline
\end{tabular}

${ }^{1}$ CCSR/NIES (Centre for Climate Research Studies Model); ${ }^{2}$ CSIROMk2 (Commonwealth Scientific and Industrial Research Organization GCM mark 2); ${ }^{3}$ CGCM2 (Canadian Global Coupled Model version 2); ${ }^{4}$ ECHAM4 (European Centre Hamburg Model version 4); ${ }^{5}$ GFDL-R30 (Geophysical Fluid Dynamics Laboratory, R-30 resolution model); ${ }^{6} \mathrm{HadCM} 3$ (Hadley Centre Coupled Model version 3); ${ }^{7}$ Multimodel ensemble.

Table 4 - World area (\%) occupied by the classes of favorability for Black Sigatoka for each month at current date and 2080 (A2 and B2 scenarios) situations predicted by six climate change models and the multimodel ensemble.

\begin{tabular}{|c|c|c|c|c|c|c|c|c|c|c|c|c|c|c|c|c|}
\hline \multirow[b]{2}{*}{ Month } & \multirow{2}{*}{\multicolumn{2}{|c|}{ Class Current }} & \multicolumn{7}{|c|}{ A2 } & \multicolumn{7}{|c|}{ B2 } \\
\hline & & & $\mathrm{CC}^{1}$ & $\mathrm{CG}^{2}$ & $\mathrm{CS}^{3}$ & $\mathrm{EC}^{4}$ & $\mathrm{GF}^{5}$ & $\mathrm{HC}^{6}$ & $\mathrm{AM}$ & $\mathrm{CC}^{1}$ & $\mathrm{CG}^{2}$ & $\mathrm{CS}^{3}$ & $\mathrm{EC}^{4}$ & $\mathrm{GF}^{5}$ & $\mathrm{HC}^{6}$ & $\mathrm{AM}$ \\
\hline \multirow{5}{*}{ January } & 1 & 0.085 & 0.003 & 0.001 & 0.002 & 0.002 & 0.001 & 0.002 & 0.001 & 0.002 & 0.003 & 0.003 & 0.005 & 0.003 & 0.002 & 0.002 \\
\hline & 2 & 4.658 & 0.603 & 0.738 & 0.770 & 0.601 & 0.821 & 0.816 & 0.772 & 0.965 & 1.463 & 1.182 & 1.034 & 1.091 & 1.243 & 1.544 \\
\hline & 3 & 2.092 & 1.983 & 1.797 & 1.771 & 1.986 & 1.721 & 1.740 & 2.661 & 1.917 & 1.344 & 1.635 & 1.813 & 1.380 & 1.576 & 2.411 \\
\hline & 4 & 6.482 & 8.138 & 7.809 & 7.800 & 7.910 & 7.786 & 7.868 & 8.636 & 8.244 & 7.818 & 7.890 & 7.946 & 7.580 & 7.848 & 8.338 \\
\hline & 5 & 86.683 & 89.274 & 89.655 & 89.657 & 89.501 & 89.671 & 89.574 & 87.929 & 88.872 & 89.372 & 89.290 & 89.202 & 89.945 & 89.331 & 87.706 \\
\hline
\end{tabular}

Continue...

Sci. Agric. (Piracicaba, Braz.), v.65, special issue, p.40-53, December 2008 
Table 4 - Continuation.

\begin{tabular}{|c|c|c|c|c|c|c|c|c|c|c|c|c|c|c|c|c|}
\hline \multirow{5}{*}{ February } & 1 & .211 & 0.001 & 0.002 & 0.003 & 0.002 & 0.002 & 0.002 & 0.002 & 0.003 & 0.010 & 0.005 & 0.007 & 0.008 & 0.007 & 0.002 \\
\hline & 2 & 4.590 & 0.508 & 0.689 & 0.683 & 0.535 & 0.811 & 0.690 & 0.705 & 0.949 & 1.467 & 1.120 & 0.921 & 1.065 & 1.109 & 1.572 \\
\hline & 3 & 1.719 & 2.155 & 1.946 & 1.897 & 2.058 & 1.767 & 1.884 & 3.187 & 2.219 & 1.636 & 1.973 & 2.165 & 1.460 & 1.962 & 3.220 \\
\hline & 4 & 7.413 & 7.899 & 7.821 & 7.780 & 7.751 & 7.751 & 7.740 & 8.669 & 8.766 & 8.624 & 8.661 & 8.645 & 7.666 & 8.609 & 8.135 \\
\hline & 5 & 86.067 & 89.437 & 89.541 & 89.637 & 89.654 & 89.669 & 89.684 & 87.437 & 88.063 & 88.263 & 88.241 & 88.262 & 89.800 & 88.312 & 87.070 \\
\hline \multirow{5}{*}{ March } & 1 & 0.207 & 0.001 & 0.003 & 0.005 & 0.002 & 0.014 & 0.003 & 0.002 & 0.005 & 0.014 & 0.010 & 0.004 & 0.024 & 0.010 & 0.007 \\
\hline & 2 & 4.666 & 0.509 & 0.658 & 0.638 & 0.500 & 0.708 & 0.630 & 0.648 & 0.783 & 1.288 & 1.028 & 0.779 & 0.955 & 1.001 & 1.306 \\
\hline & 3 & 1.396 & 2.369 & 2.213 & 2.186 & 2.306 & 2.078 & 2.187 & 3.639 & 2.718 & 2.173 & 2.416 & 2.652 & 1.778 & 2.388 & 3.843 \\
\hline & 4 & 8.274 & 9.190 & 9.119 & 9.007 & 8.925 & 8.916 & 8.914 & 9.546 & 9.407 & 9.337 & 9.305 & 9.266 & 8.852 & 9.206 & 9.120 \\
\hline & 5 & 85.457 & 87.931 & 88.007 & 88.163 & 88.267 & 88.284 & 88.266 & 86.165 & 87.087 & 87.188 & 87.242 & 87.299 & 88.390 & 87.395 & 85.724 \\
\hline \multirow{5}{*}{ April } & 1 & 0.159 & 0.001 & 0.001 & 0.001 & 0.001 & 0.001 & 0.001 & 0.000 & 0.000 & 0.002 & 0.000 & 0.000 & 0.005 & 0.000 & 0.000 \\
\hline & 2 & 4.733 & 0.364 & 0.596 & 0.590 & 0.450 & 0.614 & 0.552 & 0.568 & 0.645 & 1.296 & 0.958 & 0.707 & 0.927 & 0.820 & 0.952 \\
\hline & 3 & 1.356 & 2.761 & 2.585 & 2.580 & 2.627 & 2.492 & 2.566 & 3.351 & 3.229 & 2.556 & 2.893 & 3.016 & 2.126 & 2.942 & 3.849 \\
\hline & 4 & 8.502 & 7.954 & 7.887 & 7.816 & 7.475 & 7.307 & 7.542 & 9.344 & 7.832 & 7.613 & 7.720 & 7.348 & 7.132 & 7.486 & 9.062 \\
\hline & 5 & 85.249 & 88.920 & 88.932 & 89.013 & 89.447 & 89.586 & 89.340 & 86.737 & 88.294 & 88.533 & 88.429 & 88.930 & 89.810 & 88.753 & 86.136 \\
\hline \multirow{5}{*}{ May } & 1 & 100 & 0.013 & 0.029 & 0.036 & 0.022 & 0.033 & 0.046 & 0.013 & 0.015 & 0.046 & 0.037 & 0.018 & 0.068 & 0.039 & 0.030 \\
\hline & 2 & 4.810 & 0.327 & 0.567 & 0.578 & 0.481 & 0.529 & 0.530 & 0.602 & 0.635 & 1.499 & 1.134 & 0.843 & 0.952 & 0.868 & 1.205 \\
\hline & 3 & 1.605 & 2.697 & 2.448 & 2.415 & 2.538 & 2.473 & 2.460 & 3.209 & 2.870 & 1.937 & 2.324 & 2.626 & 1.996 & 2.573 & 3.797 \\
\hline & 4 & 8.664 & 6.833 & 6.622 & 6.569 & 6.547 & 6.565 & 6.464 & 8.778 & 7.779 & 7.462 & 7.528 & 7.526 & 6.354 & 7.404 & 8.461 \\
\hline & 5 & 84.821 & 90.131 & 90.334 & 90.402 & 90.412 & 90.400 & 90.499 & 87.398 & 88.701 & 89.055 & 88.978 & 88.986 & 90.630 & 89.116 & 86.508 \\
\hline \multirow{5}{*}{ June } & 1 & 0.067 & 0.047 & 0.072 & 0.085 & 0.051 & 0.074 & 0.084 & 0.039 & 0.054 & 0.064 & 0.063 & 0.051 & 0.093 & 0.063 & 0.039 \\
\hline & 2 & 4.806 & 0.645 & 0.950 & 0.964 & 0.776 & 0.882 & 0.809 & 1.049 & 1.163 & 2.028 & 1.707 & 1.260 & 1.405 & 1.266 & 1.859 \\
\hline & 3 & 3.027 & 3.051 & 2.715 & 2.653 & 2.887 & 2.722 & 2.787 & 3.687 & 3.018 & 2.078 & 2.413 & 2.880 & 2.143 & 2.838 & 3.664 \\
\hline & 4 & 7.416 & 7.757 & 6.423 & 7.480 & 6.518 & 6.176 & 6.459 & 8.484 & 7.813 & 6.891 & 7.627 & 7.025 & 6.000 & 6.881 & 8.543 \\
\hline & 5 & 84.683 & 88.499 & 89.839 & 88.818 & 89.769 & 90.146 & 89.861 & 86.741 & 87.952 & 88.939 & 88.189 & 88.785 & 90.359 & 88.952 & 85.896 \\
\hline \multirow{5}{*}{ July } & 1 & 0.008 & 0.047 & 0.079 & 0.083 & 0.060 & 0.086 & 0.079 & 0.053 & 0.077 & 0.072 & 0.074 & 0.070 & 0.083 & 0.067 & 0.068 \\
\hline & 2 & 4.489 & 1.082 & 1.827 & 1.654 & 1.380 & 1.622 & 1.386 & 1.737 & 2.146 & 2.819 & 2.557 & 2.338 & 2.134 & 2.243 & 2.993 \\
\hline & 3 & 3.905 & 3.702 & 2.868 & 3.099 & 3.448 & 3.128 & 3.379 & 4.066 & 3.468 & 2.682 & 2.995 & 3.251 & 2.540 & 3.314 & 3.677 \\
\hline & 4 & 9.603 & 9.479 & 8.348 & 7.441 & 7.758 & 7.762 & 8.012 & 11.615 & 11.200 & 8.975 & 8.907 & 9.317 & 6.959 & 9.350 & 10.587 \\
\hline & 5 & 81.995 & 85.690 & 86.878 & 87.723 & 87.354 & 87.402 & 87.144 & 82.529 & 83.109 & 85.452 & 85.468 & 85.024 & 88.284 & 85.026 & 82.674 \\
\hline \multirow{5}{*}{ August } & 1 & 0.009 & 0.027 & 0.053 & 0.056 & 0.030 & 0.055 & 0.053 & 0.031 & 0.049 & 0.044 & 0.044 & 0.032 & 0.055 & 0.040 & 0.026 \\
\hline & 2 & 4.949 & 1.039 & 1.718 & 1.652 & 1.264 & 1.526 & 1.434 & 1.811 & 2.293 & 2.781 & 2.480 & 2.196 & 2.033 & 2.397 & 3.061 \\
\hline & 3 & 3.725 & 4.216 & 3.152 & 3.302 & 3.756 & 3.438 & 3.526 & 4.104 & 3.492 & 2.841 & 3.194 & 3.522 & 2.833 & 3.306 & 3.658 \\
\hline & 4 & 9.718 & 13.150 & 8.719 & 9.975 & 10.331 & 9.698 & 11.002 & 13.101 & 12.977 & 8.857 & 10.593 & 10.650 & 8.497 & 10.530 & 10.936 \\
\hline & 5 & 81.599 & 81.569 & 86.358 & 85.014 & 84.618 & 85.283 & 83.986 & 80.954 & 81.190 & 85.478 & 83.689 & 83.600 & 86.582 & 83.726 & 82.319 \\
\hline \multirow{5}{*}{ September } & 1 & 0.006 & 0.017 & 0.017 & 0.015 & 0.011 & 0.011 & 0.013 & 0.007 & 0.011 & 0.010 & 0.011 & 0.008 & 0.021 & 0.011 & 0.006 \\
\hline & 2 & 4.794 & 0.681 & 1.016 & 1.082 & 0.841 & 0.997 & 1.023 & 1.053 & 1.499 & 1.779 & 1.587 & 1.331 & 1.347 & 1.544 & 1.739 \\
\hline & 3 & .371 & 2.924 & 2.481 & 2.438 & 2.708 & 2.506 & 2.518 & 3.068 & 2.504 & 2.130 & 2.340 & 2.625 & 2.124 & 2.382 & 2.621 \\
\hline & 4 & 142 & 8.656 & 7.458 & 7.688 & 7.983 & 7.476 & 7.842 & 10.197 & 9.486 & 8.624 & 8.890 & 9.076 & 7.344 & 8.814 & 9.797 \\
\hline & 5 & 83.686 & 87.722 & 89.029 & 88.777 & 88.457 & 89.010 & 88.604 & 85.676 & 86.500 & 87.457 & 87.171 & 86.960 & 89.163 & 87.249 & 85.837 \\
\hline \multirow{5}{*}{ October } & 1 & 0.006 & 0.001 & 0.001 & 0.001 & 0.001 & 0.001 & 0.001 & 0.000 & 0.002 & 0.004 & 0.002 & 0.002 & 0.003 & 0.003 & 0.002 \\
\hline & 2 & 4.523 & 0.380 & 0.708 & 0.707 & 0.503 & 0.664 & 0.624 & 0.660 & 0.786 & 1.351 & 1.094 & 0.883 & 1.063 & 1.070 & 1.077 \\
\hline & 3 & 1.496 & 2.438 & 2.046 & 2.056 & 2.252 & 2.079 & 2.145 & 2.411 & 2.184 & 1.517 & 1.843 & 2.038 & 1.623 & 1.874 & 2.149 \\
\hline & 4 & 7.621 & 7.525 & 6.522 & 6.715 & 6.674 & 6.591 & 6.930 & 8.266 & 7.839 & 6.782 & 7.222 & 7.127 & 5.974 & 7.325 & 7.603 \\
\hline & 5 & 86.354 & 9.656 & 90.724 & 90.522 & 90.570 & 90.665 & 90.300 & 88.662 & 89.189 & 90.347 & 89.838 & 89.950 & 91.337 & 89.729 & 89.168 \\
\hline \multirow{5}{*}{ November } & 1 & 022 & 0.005 & 002 & 0.002 & 0.002 & 0.002 & 0.004 & 0.001 & 0.003 & 0.005 & 0.003 & 0.004 & 0.008 & 0.005 & 0.004 \\
\hline & 2 & 4.112 & 0.514 & 0.847 & 0.875 & 0.721 & 0.862 & 0.833 & 0.857 & 1.061 & 1.459 & 1.245 & 1.115 & 1.193 & 1.230 & 1.519 \\
\hline & 3 & 1.516 & 2.298 & 1.942 & 1.917 & 2.060 & 1.928 & 1.940 & 2.222 & 1.986 & 1.557 & 1.786 & 1.914 & 1.566 & 1.790 & 2.033 \\
\hline & 4 & 7.375 & 6.003 & 5.565 & 5.621 & 5.675 & 5.656 & 5.721 & 7.674 & 6.111 & 5.760 & 5.930 & 5.887 & 5.376 & 5.920 & 7.893 \\
\hline & 5 & 86.975 & 91.181 & 91.644 & 91.585 & 91.542 & 91.552 & 91.501 & 89.246 & 90.839 & 91.219 & 91.036 & 91.082 & 91.857 & 91.054 & 88.551 \\
\hline \multirow{5}{*}{ December } & 1 & & & & 0.005 & 0.002 & 0.002 & & & & 0.007 & 0.006 & 0.007 & 0.006 & 0.002 & 0.005 \\
\hline & 2 & 4.269 & & 0.870 & 0.898 & 0.758 & 0.892 & 0.909 & 0.865 & 1.020 & 1.487 & 1.228 & 1.108 & 1.071 & 1.246 & 1.425 \\
\hline & 3 & 1.795 & 2.166 & 1.942 & 1.912 & 2.069 & 1.926 & 1.904 & 2.218 & 1.991 & 1.437 & 1.753 & 1.877 & 1.717 & 1.723 & 2.291 \\
\hline & 4 & 7.480 & 6.100 & 5.655 & 5.818 & 5.893 & 5.862 & 5.865 & 8.496 & 8.010 & 7.573 & 7.804 & 7.747 & 5.603 & 7.718 & 8.727 \\
\hline & 5 & 86.407 & 91.064 & 91.532 & 91.367 & 91.278 & 91.317 & 91.317 & 88.419 & 88.974 & 89.495 & 89.209 & 89.261 & 91.603 & 89.311 & 87.552 \\
\hline
\end{tabular}

${ }^{1}$ CCSR/NIES (Centre for Climate Research Studies Model); ${ }^{2}$ CSIROMk2 (Commonwealth Scientific and Industrial Research Organization GCM mark 2); ${ }^{3}$ CGCM2 (Canadian Global Coupled Model version 2); ${ }^{4}$ ECHAM4 (European Centre Hamburg Model version 4); ${ }^{5}$ GFDL-R30 (Geophysical Fluid Dynamics Laboratory, R-30 resolution model); ${ }^{6} \mathrm{HadCM} 3$ (Hadley Centre Coupled Model version 3); ${ }^{7}$ Multimodel ensemble. 


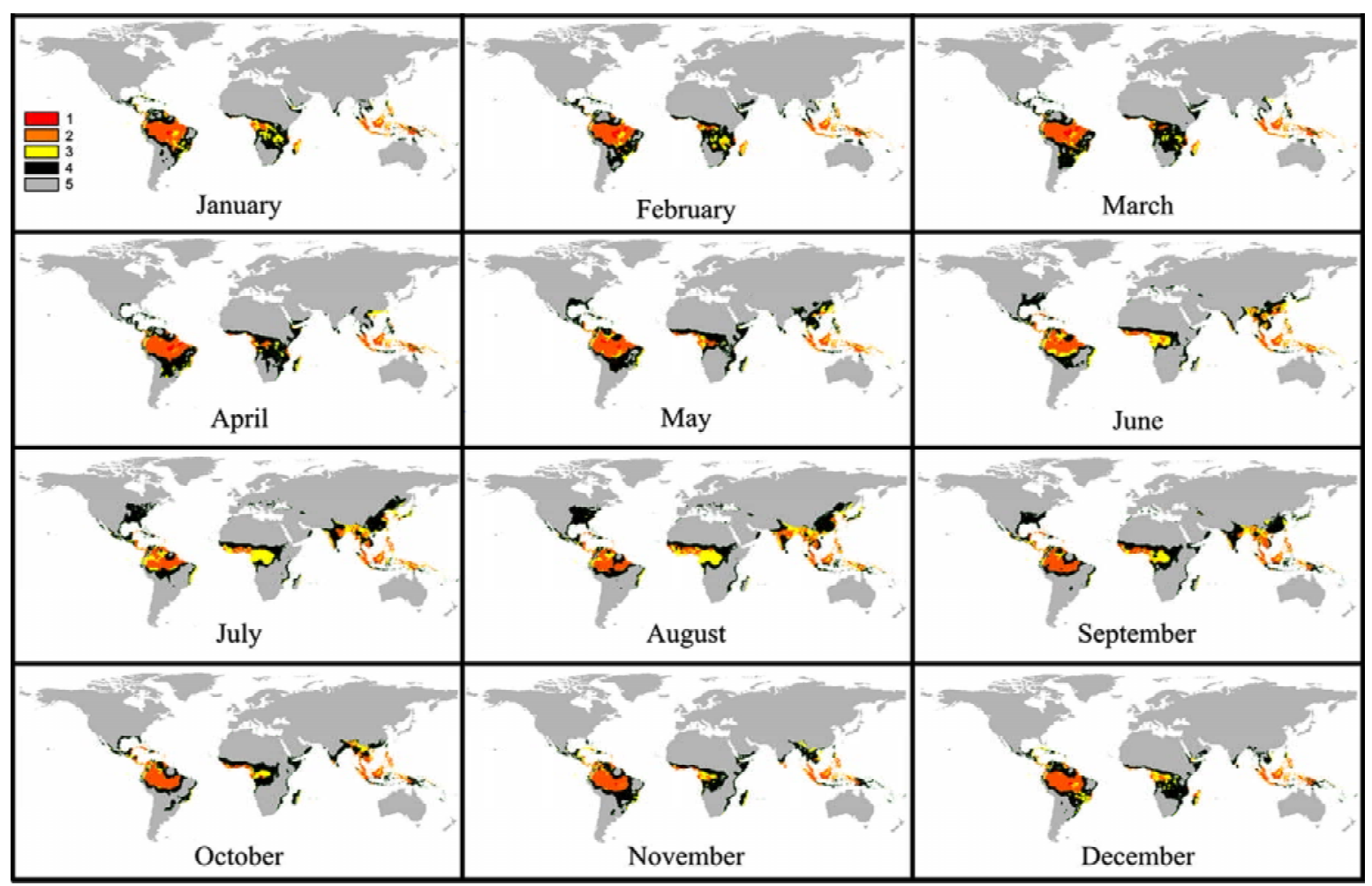

Figure 1- Maps representing current (1961-1990 average) worldwide spatial distribution of the classes of favorabilityfor Black Sigatoka (BS) for January to December, where Class 1: highly favorable to BS; Class 2: favorable to BS; Class 3: relatively favorable to BS; Class 4: little favorable to BS and Class 5: unfavorable to BS.

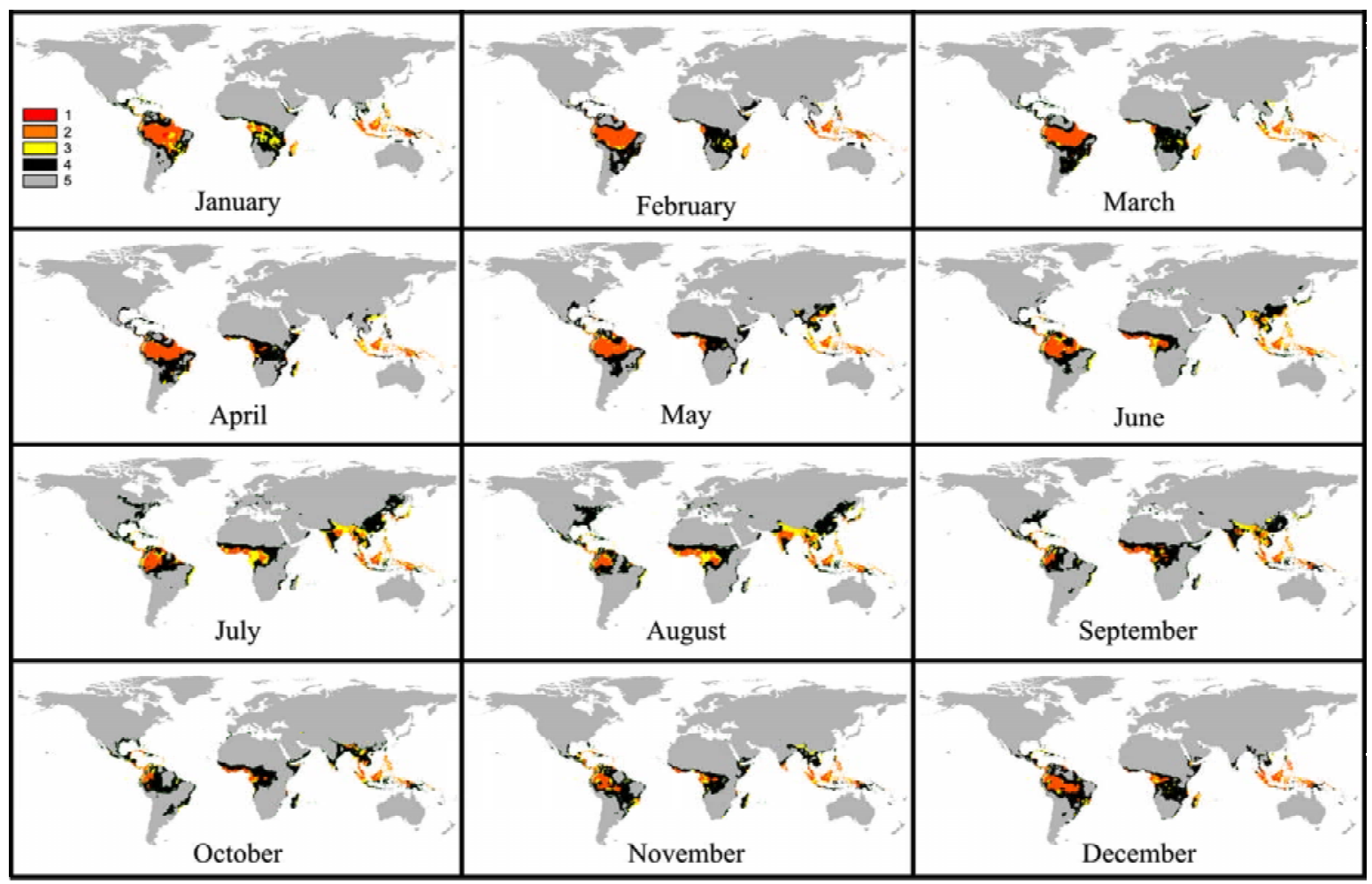

Figure 2 - Maps representing future worldwide spatial distribution of the classes of favorability for Black Sigatoka (BS), predicted by the use of the "multimodel emsemble", for January to December, for the decade of 2020 (A2 scenario), where Class 1: highly favorable to BS; Class 2: favorable to BS; Class 3: relatively favorable to BS; Class 4: little favorable to BS and Class 5: unfavorable to BS. 


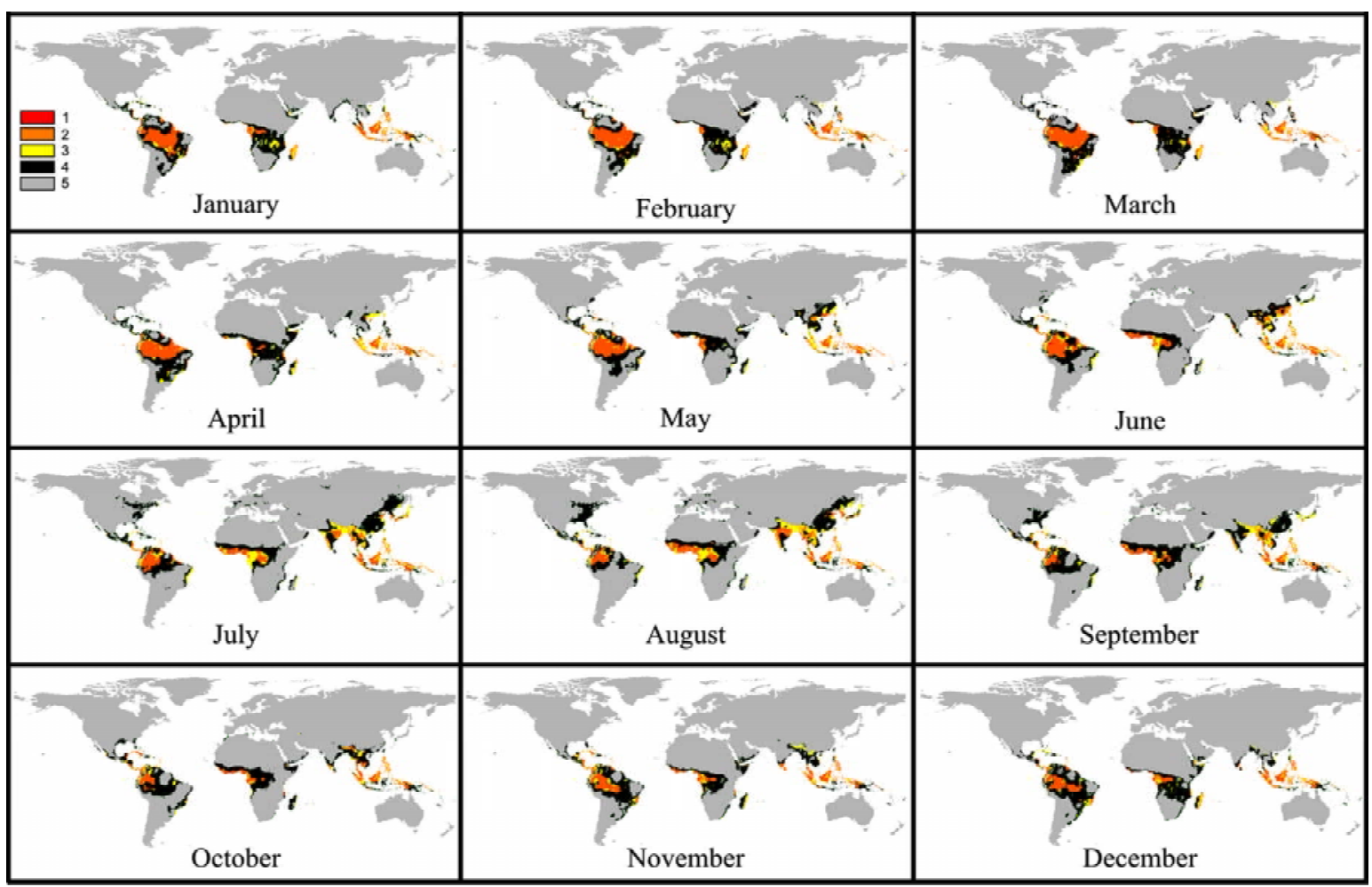

Figure 3 - Maps representing future worldwide spatial distribution of the classes of favorability for Black Sigatoka (BS), predicted by the use of the "multimodel emsemble", for January to December, for the decade of 2020 (B2 scenario), where Class 1: highly favorable to BS; Class 2: favorable to BS; Class 3: relatively favorable to BS; Class 4: little favorable to BS and Class 5: unfavorable to BS.

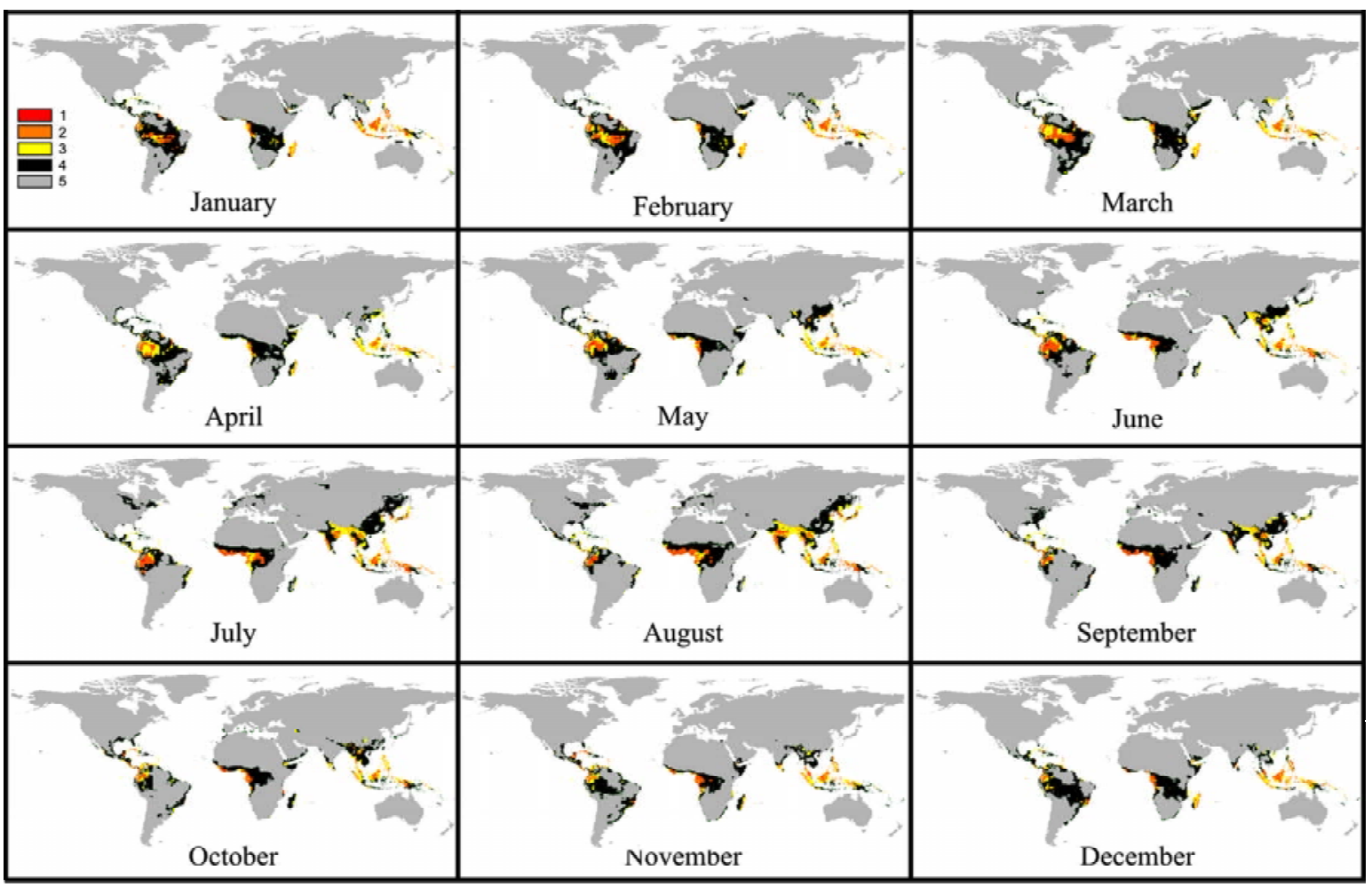

Figure 4 - Maps representing future worldwide spatial distribution of the classes of favorability for Black Sigatoka (BS), predicted by the use of the "multimodel emsemble", for January to December, for the decade of 2050 (A2 scenario), where Class 1: highly favorable to BS; Class 2: favorable to BS; Class 3: relatively favorable to BS; Class 4: little favorable to BS and Class 5: unfavorable to BS. 


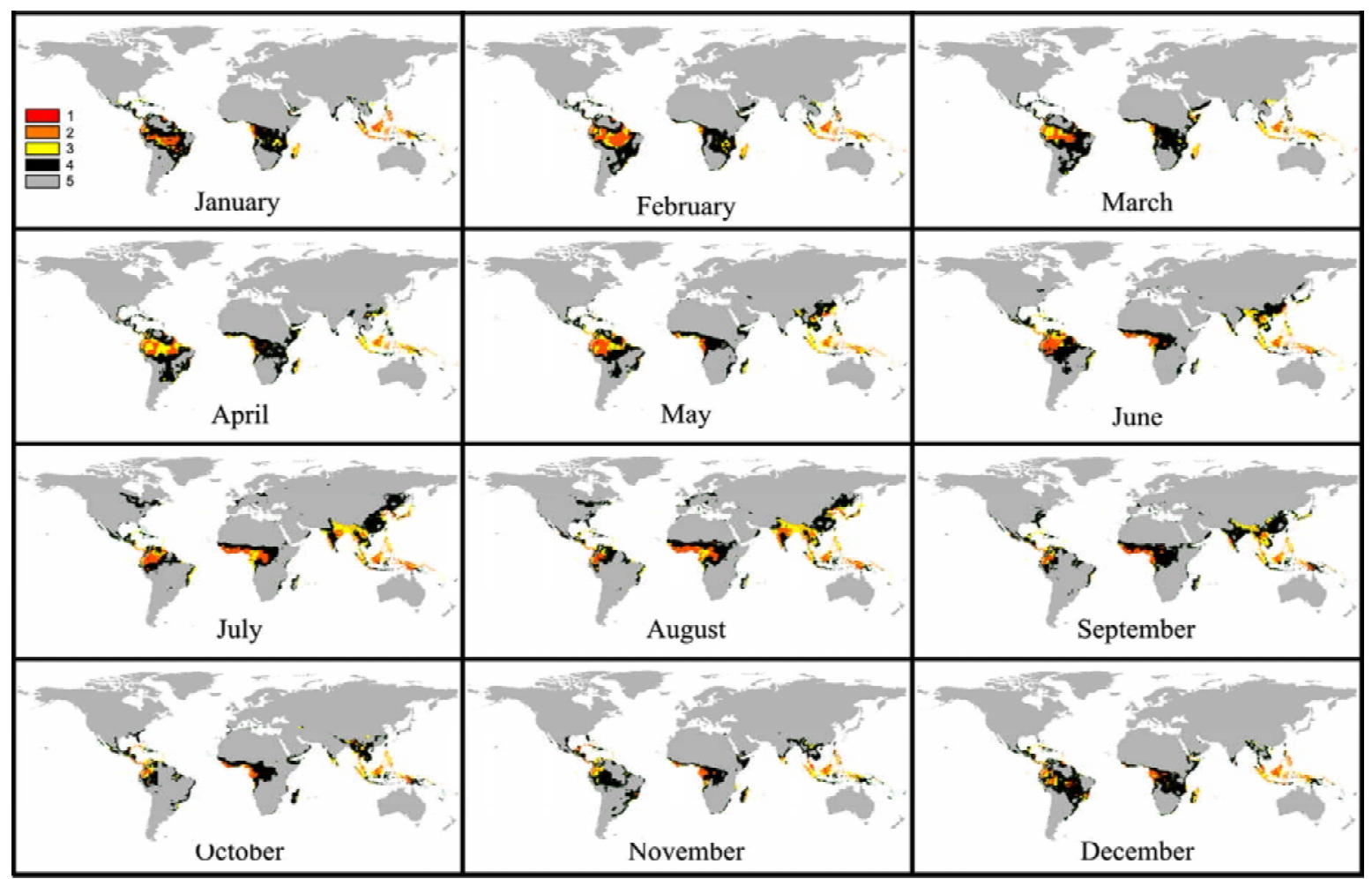

Figure 5 - Maps representing future worldwide spatial distribution of the classes of favorability for Black Sigatoka (BS), predicted by the use of the "multimodel emsemble", for January to December, for the decade of 2050 (B2 scenario), where Class 1: highly favorable to BS; Class 2: favorable to BS; Class 3: relatively favorable to BS; Class 4: little favorable to BS and Class 5: unfavorable to BS.

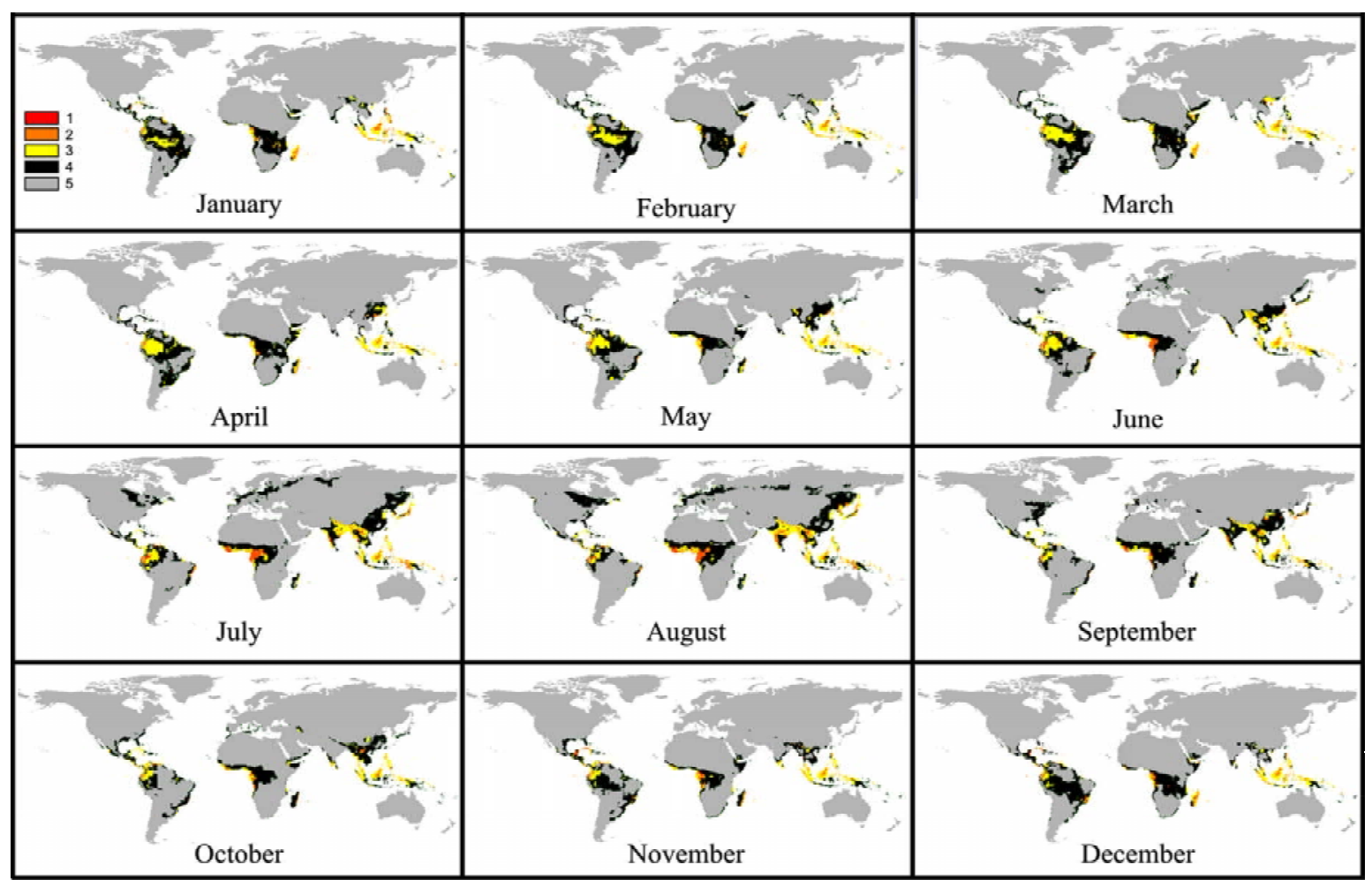

Figure 6 - Maps representing future worldwide spatial distribution of the classes of favorability for Black Sigatoka (BS), predicted by the use of the "multimodel emsemble", for January to December, for the decade of 2080 (A2 scenario), where Class 1: highly favorable to BS; Class 2: favorable to BS; Class 3: relatively favorable to BS; Class 4: little favorable to BS and Class 5: unfavorable to BS. 


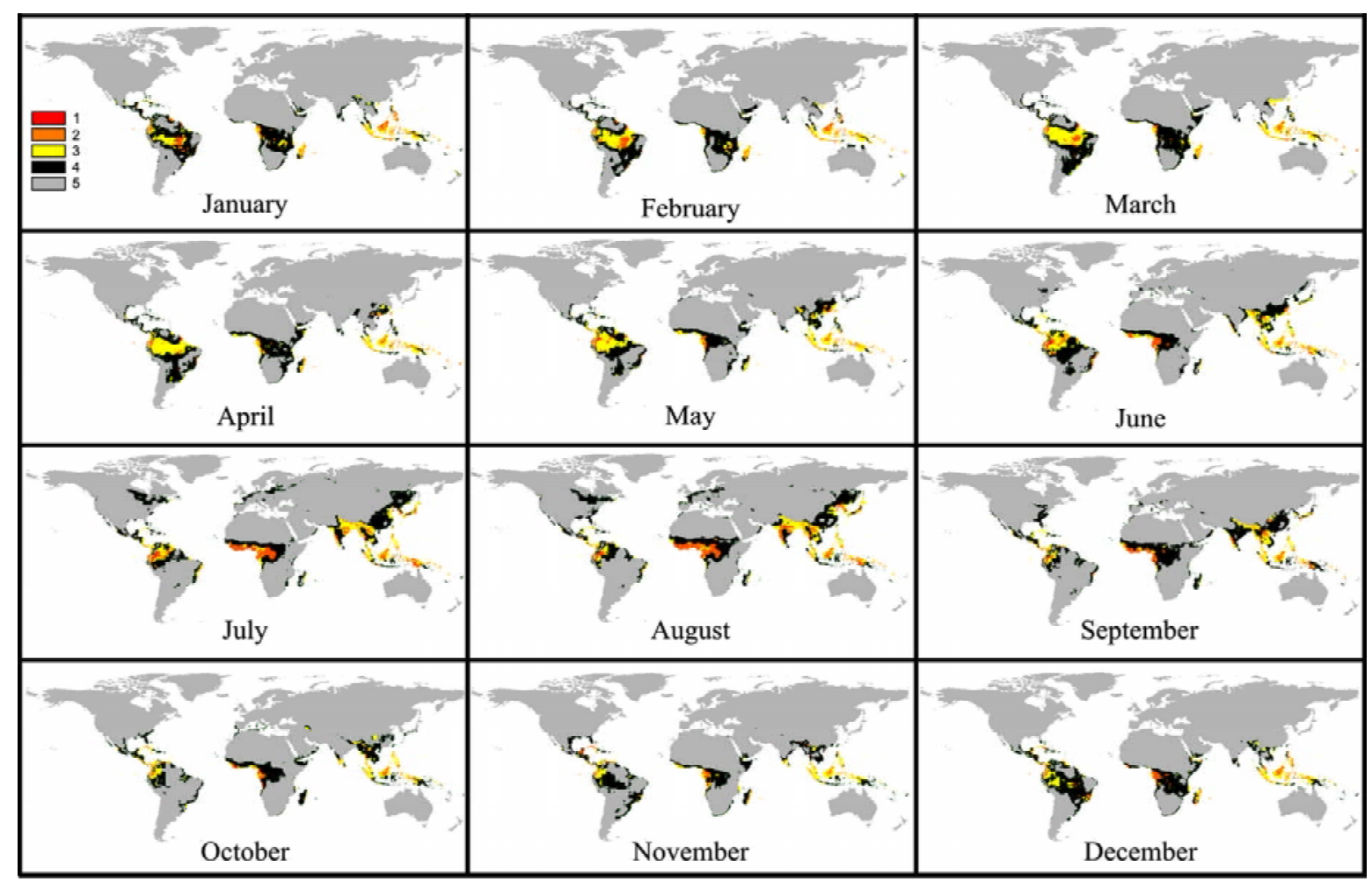

Figure 7 - Maps representing future worldwide spatial distribution of the classes of favorability for Black Sigatoka (BS), predicted by the use of the "multimodel emsemble", for January to December, for the decade of 2080 (B2 scenario), where Class 1: highly favorable to BS; Class 2: favorable to BS; Class 3: relatively favorable to BS; Class 4: little favorable to BS and Class 5: unfavorable to BS.

where they are disseminated by air currents (Meredith et al., 1973). Conidia germinate over a wider range of relative humidity (92 to $100 \%$ ) as compared to ascospores (98 to 100\%). The effect of temperature on the germination can be characterized by a quadratic response function, with an estimated optimum at $26.5^{\circ} \mathrm{C}$. Stover (1983) observed maximum growth of ascospore germ tubes at 26 to $28^{\circ} \mathrm{C}$ after $24 \mathrm{~h}$ incubation. The optimal temperature range for disease development was $25-28^{\circ} \mathrm{C}$ (Jacome et al., 1991; Jacome \& Schuh, 1992).

Regarding the worldwide geographical distribution of the disease considering current and future periods, the results suggested that for both scenarios (A2 and B2) there will be a decrease in areas classified as 1 (highly favorable), 2 (favorable) and 3 (relatively favorable) and an increase of areas classified at 4 (little favorable) and 5 (unfavorable) (Figures 1 to 7) (Tables 2 to 4). These changes will be gradual for the decades of 2020, 2050 and 2080, with some exceptions that will be presented later in this paper. Results are in agreement with Ghini et al. (2007) who predicted that there will be a reduction of the favorable area to BS in Brazil in the future. Other authors also showed changes on the spatial distribution of favorability classes for other pathosystems in the fu- ture, like Carter et al. (1996), Boag et al. (1991), Brasier \& Scott (1994) and Brasier et al. (1996).

Variation in the worldwide geographical distribution of the disease will be higher for the A2 scenario as compared to $\mathrm{B} 2$, and will be different for the decades of 2020, 2050 and 2080 (Figures 1 to 7 and Tables 2 to 4). These findings are consistent with those of Ghini et al. (2007) on the probable impacts of climatic changes on BS in Brazil. The authors concluded that there will be a reduction in the favorable area to the disease in the Brazil and that the reduction will be higher for A2 scenario than for B2.

Some exceptions were detected, for example classes 3 and 4 in 2080. For class 3, an increase was observed in the area occupied by the same for scenario A2, which probably is related to the fact that some world areas that are not so warm (e.g. subtropical areas) will become favorable to the disease with an increase in temperature, especially when considering the interval between $20-25^{\circ} \mathrm{C}$.

In the case of class 4 , a decrease was also observed in the area represented by the same for the A2 scenario in 2080 which could be explained by a greater decrease in relative humidity for this decade. Gauhl (1994) reported that there is a reduction in the production of inoculum of $M$. fijiensis during drier (or 
less rainy) months of the year in the Caribbean zone of Costa Rica, which is also the season with the lowest temperature. Others studies pointed out that successful infection is promoted by extended periods of high humidity and the presence of free water on leaves (Mayorga, 1990; Fullerton, 1994).

Analysis of the geographical distribution of the disease among months showed that in the future there will be a displacement in the worldwide geographical distribution of BS (Figures 1 to 7) in which unfavorable areas could become favorable and vice-versa as a function of increases in temperature and/or decreases in relative humidity. Colder months in the southern hemisphere (June, July and August), which are considered less favorable for the disease development at the current climatic conditions, could become more favorable due to increases in temperature. On the other hand, months which presented higher temperatures in this hemisphere (November, December and January) could become unfavorable to BS because of the extreme increases in the temperature and/or decreases in relative humidity. A great reduction in the areas of classes 1 (highly favorable), 2 (favorable) and 3 (relatively favorable) in November and December in the southern hemisphere will probably occur. This reduction will likely be lower in June and July. Thus, favorability will probably be gradually displaced from November to May (current condition) to January to July, for the years 2020 to 2080 .

On the other hand, no such displacement was evident in the northern hemisphere for the classes among months of the year, due to hemispheric asymmetries in climate change predictions. Months most likely favorable to disease development will continue to be May to October. Additionally, it was clear from the results that areas occupied by classes 1, 2, 3 and 4 of the aforementioned months will increase, particularly in Southeast Asia, India and center-east United States. Thus, conditions of favorability to BS will be displaced northward.

When we consider three main worldwide banana producers (FAO, 2007) it is possible to conclude that spatial distribution of Black Sigatoka will be different in each country. For example, in the case of Brazil a gradual reduction in the favorable area to BS probably will occur in the decades of 2020, 2050 and 2080. However, favorable areas to the disease will probably increase in India and China if IPCC scenarios remain consistent.

Information available in the literature could help us to explain differences that will occur with spatial distribution of BS in the future. Simulations carried out in this study showed that the class 4 of favorability to disease development in Brazil tends to increase. This will be a function of a decrease in relative humidity which could increase incubation and latent periods (lower numbers of pathogen cycles per year). Thus, the importance of the disease will probably be lower. In India and China, the situation will be the inverse.

Disease development is strongly related to weather conditions and plant susceptibility. Under very favorable conditions in Costa Rica and with a susceptible host, incubation periods of $M$. fijiensis can be as short as 13 to 14 days, whereas during periods of unfavorable weather, the duration of the incubation period can extend up to 35 days (Marín et al, 2003). Similar reports exist for Nigeria on plantains (Mobambo et al., 1996). During the rainy season, the incubation period was 14 days but in the dry season 24 days. Differences in the latent period from December 1993 to May 1995 for the susceptible cultivar Grande Nine, which is widely used for the fresh banana market, were observed in Guapiles, Costa Rica (Marín et al., 2003). The latent period ranged from 25 days during the rainy season (June to December) to 70 days during the dry season at the same locality. When the weather is highly conducive for ascospore discharge and infection, many infections occur on the leaves. When infections are dense, they rapidly coalesce at a very early stage of development, accelerating the appearance of mature spots that are characterized by the presence of pseudothecia and ascospores (Fouré et al., 1984). Under theses conditions, leaves are rapidly and severely damaged.

In a general way, the use of the "multimodel ensemble" allowed reduction in the variability of simulation compared with data estimated by each model individually (Tables 2 to 4). It is important to point out that the "multimodel ensemble" analysis was used with success in other studies (Bergot et al., 2004; Cerri et al., 2007; Ghini et al., 2007; Marengo, 2007), not only related to Black Sigatoka or plant diseases. The adopted methodology allows the comparison between results obtained by each model and by the average of all ("multimodel ensemble"). This comparison could be extremely useful to direct and help new researches about the use of climate change models on the spatial distribution of plant diseases.

However, some exceptions were observed, particularly in 2080, in which each model simulated a decrease in the area occupied by classes considered favorable for disease development while results generated by the use of the "multimodel ensemble" simulated an increase (Tables 2 to 4). For the A2 scenario in 2080, great variations in the way data were analyzed were observed particularly for classes 3 (January and July) and 4 (April, May, July, September, October, November and December). For the B2 scenario for the 
same decade variations were detected for the results of classes 3 (January and June) and 4 (April and November). Thus, caution should be taken when using the "multimodel ensemble". More studies are necessary to reduce these uncertainties.

Climate changes certainly will affect the development of the plant. For the current conditions, banana is cultivated in more than 100 countries throughout the world, however, in the future changes in weather conditions may result in some areas becoming unsuitable for banana cultivations, causing commercial changes. Such situation, however, can be prevented with genetic improvement. In the same way, the pathogen can suffer more selection pressure. Prediction of the effect of climate change on pathogens and plants is difficult and speculative because the magnitude and range of these changes is very uncertain (Boag et al., 1991; Hillier, 1993). Nevertheless, although speculative, published data has suggested potential problems that may exist under a modified climate (IPCC, 2007).

Most plant disease models use different weather variables and operate at different spatial and temporal scales than do the global climate models. Improvements in the methodology are necessary to realistically assess disease impacts at a global scale.

Experimental research on a diverse range of disease systems is necessary to improve comprehension of climate change impacts. Given the multitude of atmospheric and climatic factors, the possible change in scenarios and the number of disease systems, modeling approaches to impact assessment need to be strengthened. For instance, changes in both mean temperature and its variability are equally important in predicting the potential impact of climate change (Scherm \& Bruggen, 1994). Given that climate change is a global issue, the focus needs to shift from paddock-based assessment on specific diseases to a more ecologically relevant spatial unit (Scherm et al., 2000) to consider climate with other associated changes in land use and vegetation cover (Luo et al., 1995), among others.

Results obtained in this study could help to answer two important questions: (i) could preventative measures delay or prevent further spread of the disease?; and (ii) are those countries threatened by the disease taking measures to prevent the entry of the pathogen?

\section{ACKNOWLEDGEMENTS}

To CNPq (project 473999/2006-4), and for first author's support also by CNPq (project 308596/ 2006-4).

\section{REFERENCES}

BERGOT, M.; CLOPPET, E.; PÉRARNAUD, V.; DÉQUÉ, M.; MARÇAIS, B.; DESPREZ-LOUSTAU, M.L. Simulation of potential range expansion of oak disease caused by Phytophthora cinnamomi under climate change. Global Change Biology, v.10, p.1539-1552, 2004.

BOAG, B.; CRAWFORD, J.W.; NEILSON, R. The effect of potential climatic changes on the geographical distribution of the plantparasitic nematodes Xiphinema and Longidorus in Europe. Nematologica, v.37, p.312-323, 1991.

BOURGEOIS, G.; BOURQUE, A.; DEAUDELIN, G. Modelling the impact of climate change on disease incidence: a bioclimatic challenge. Canadian Journal of Plant Pathology, v.26, p.284-290, 2004.

BRASIER, C.M.; DREYER, E.; AUSSENAC, G. Phytophthora cinnamomi and oak decline in southern Europe. Environmental constraints including climate change. Annales des Sciences Forestieres, v.53, p.347-358, 1996.

BRASIER, C.M.; SCOTT, J.K. European oak declines and global warming: a theoretical assessment with special reference to the activity of Phytophthora cinnamomi. Bulletin OEPP/EPPO Bulletin, v.24, p.221-232, 1994.

CARLIER, J.; FOURÉ, E.; GAUHL, F.; JONES, D.R.; LEPOIVRE, P.; MOURICHON, X.; PASBERG-GAUHL, C.; ROMERO, R.A. Black leaf streak. In: JONES, D.R. (Ed.) Diseases of banana, abacá and enset. Wallingford: CAB International, 2000. p.37-79.

CARTER, T.R.; SAARIKKO, R.A.; NIEMI, K.J. Assessing the risks and uncertainties of regional crop potential under a changing climate in Finland. Agricultural and Food Science in Finland, v.5, p.329-350, 1996.

CERRI, C.E.P.; SPAROVEK, G.; BERNOUX, M.; EASTERLING, W.E.; MELILLO, J.M.; CERRI, C.C. Tropical agriculture and global warming: impacts and mitigation options. Scientia Agricola, v.64, p.83-99, 2007.

CHAKRABORTY, S.; TIEDEMANN, A.V.; TENG, P.S. Climate change: potential impact on plant diseases. Environmental Pollution, v.108, p.317-326, 2000.

CHAKRABORTY, S.; MURRAY, G.M.; MAGAREY, P.A.; YONOW, T.; SIVASITHAMPARAM, K.; O'BRIEN, R.G.; CROFT, B.J.; BARBETTI, M.J.; OLD, K.M.; DUDZINSKI, M.J.; SUTHERST, R.W.; PENROSE, L.J.; ARCHER, C.; EMMETT, R.W. Potential impact of climate change on plant diseases of economic significance to Australia. Australasian Plant Pathology, v.27, p.15-35, 1998.

FOOD AND AGRICULTURE ORGANIZATION - FAO. ProdSTAT. Available at: http://faostat.fao.org. Accessed 30 Jul. 2007.

FOURÉ, E.; GRISONI, M.; ZURFLUH, R. Les cercosporiosis du bananier et leurs traitements. Comportemente des variétés. Etude de la sensibilité variétale des bananiers et plantains B Mycosphaerella fijiensis Morelet et de quelques caractéristiques biologiques de la maladie de rales noires au Gabon. Fruits, v.39, p.365-377, 1984.

FULLERTON, R.A. Sigatoka leaf diseases. In: PLOETZ, R.C.; ZENTMYER, G.A.; NISHIJINIA, W.T.; ROHRBACH, K.G.; OHR, H.D. (Ed.) Compendium of tropical fruit diseases. St Paul: American Phytopathological Society, 1994. p.12-14.

GAUHL, F. Epidemiology and ecology of Black Sigatoka (Mycosphaerella fijiensis Morelet) on plantain and banana (Musa spp.) in Costa Rica, Central America. Montpellier: INIBAP, 1994. 120p.

GHINI, R.; HAMADA, E.; GONÇALVES, R.R.V.; GASPAROTTO, L.; PEREIRA, J.C. Análise de risco das mudanças climáticas globais sobre a sigatoka-negra da bananeira no Brasil. Fitopatologia Brasileira, v.32, p.197-204, 2007.

HAMADA, E.; GHINI, R.; GONÇALVES, R.R.V. Efeito da mudança climática sobre problemas fitossanitários de plantas: metodologia de elaboração de mapas. Engenharia Ambiental, v.3, p.73$85,2006$. 
HILLIER, S. Climate change impacts on British vegetation creating tomorrow's climate today. NERC News, v.26, p.27-29, 1993.

IPCC. Climate change 2007: the physical science basis: summary for policymakers. Geneva: IPCC, 2007. 18p. Available at: http:/ /www.ipcc.ch/SPM2feb07.pdf. Accessed 10 Jun. 2007.

IPCC. Climate change 2001: the scientific basis: summary for policymakers. Geneva: IPCC, 2001. 20p. Available at: http:// www.ipcc.ch/pub/spm22-01.pdf. Accessed 10 Jun. 2007.

JACOME, L.H.; SCHUH, W. Effects of leaf wetness duration and temperature of development of black Sigatoka disease on banana infected by Mycosphaerella fijiensis var. difformis. Phytopathology, v.82, p.515-520, 1992.

JACOME, L.H.; SCHUH, W.; STEVENSON, R.E. Effect of temperature and relative humidity on germination and germ tube development of Mycosphaerella fijiensis var. difformis. Phytopathology, v.12, p.1480-1485, 1991.

LUO, Y.; TEBEEST, D.O.; TENG, P.S.; FABELLAR, N.G. Simulation studies on risk analysis of rice leaf blast epidemics associated with global climate change in several Asian countries. Journal of Biogeography, v.22, p.673-678, 1995.

MAYORGA, M.H. La Raya negra (Mycosphaerella fijiensis Morelet) del plátano y del banano. I. Ciclo de vida del patógeno bajo las condiciones del Urabá. Revista ICA, v.25, p.69-77, 1990.

MARENGO, J. Cenários de mudanças climáticas para o Brasil em 2100. Ciência \& Ambiente, v.34, p.97-114, 2007.

MARÍN, D.H.; ROMERO, R.A.; GUZMÁN, M.; SUTTON, T.B. Black Sigatoka: an increasing threat to banana cultivation. Plant Disease, v.87, p.208-222, 2003.

MEREDITH, D.S.; LAWRENCE, J.S.; FIRMAN, I.D. Ascospore release and dispersal on black leaf streak disease of bananas (Mycosphaerella fijiensis). Transactions of the British Mycological Society, v.60, p.547-554, 1973.

MOBAMBO, K.M.; GAUHL, F.; PASBERG-GAUHL, C.; ZUOFA, K. Season and plant age effects evaluation of plantain for response to Black Sigatoka disease. Crop Protection, v.15, p.609-614, 1996.
MOULIOM-PEFOURA, A.; LASSOUDIÈRE, A.; FOKO, J.; FONTEM, D.A. Comparison of development of Mycosphaerella fijiensis and Mycosphaerella musicola on banana and plantain in various ecological zones in Cameroon. Plant Disease, v.80, p.950-954, 1996

MOURICHON, X.; CARLIER, J.; FOURÉ, J. Sigatoka leaf spot diseases. Montpellier: INIBAP, 1997. 4p. (Musa Disease Fact Sheet, 8).

NEW, M.; LISTER, D.; HULME, M.; MAKIN, I. A high-resolution data set of surface climate over global land areas. Climate Research, v.21, p.1-25, 2002.

ROMERO, R.A.; SUTTON, T.B. Reaction of four Musa genotypes at three temperatures to isolates of Mycosphaerella fijiensis from different geographical regions. Plant Disease, v.81, p.1139-1142, 1997

SCHERM, H. Climate change: can we predict the impacts on plant pathology and pest management? Canadian Journal of Plant Pathology, v.26, p.267-273, 2004.

SCHERM, H.; SUTHERST, R.W.; HARRINGTON, R.; INGRAM, J.S.I. Global networking for assessment of impacts of global change on plant pests. Environmental Pollution, v.108, p.333-341, 2000.

SCHERM, H.; BRUGGEN, A.H.C. van. Global warming and nonlinear growth: how important are changes in average temperature? Phytopathology, v.84, p.1380-1384, 1994.

STOVER, R.H. The effect of temperature on ascospore germ tube growth of Mycosphaerella musicola and Mycosphaerella fijiensis var. difformis. Fruits, v.38, p.625-628, 1983.

Received November 01, 2007

Accepted June 12, 2008 